\title{
The Second-Tier Trap: Theory and Experimental Evidence
}

\author{
Duk Gyoo Kim*
}

Current version: April 6, 2017

\begin{abstract}
Winner-take-all competitions can lead to the person in the second-tier (middle-tier) environment having the worst expected payoff when players exclusively choose their environment and exert effort before their random, heterogeneous environmental supports are realized. The tiers are defined by the ranks in pairwise competitions. The second-tier trap (STT) is a situation in which a player from the second-tier environment has the worst expected payoff even though his expected environmental support is strictly greater than that of the third-tier player. A sufficient condition for the STT is that the ex-ante advantages, the winning probabilities when all the players exert the same amount of effort regardless of their environment, be the same for those two environments. I claim that this sufficient condition for the STT is so weak that players can easily be tempted to choose the second-tier environment, which is the wrong decision. Lab experiments strongly support this claim.
\end{abstract}

Key words contests, all-pay auction, Lazear-Rosen model, laboratory experiments JEL classification C72, C91, D81

\section{Introduction}

I consider a winner-take-all competition among $n$ players in which their "environmental supports" are random and heterogeneous. In the first phase, players exclusively choose an

${ }^{*}$ Division of the Humanities and Social Sciences, California Institute of Technology, Pasadena, CA, USA. Email: dgkim@caltech.edu. I would especially like to thank an anonymous referee, Robert H. Frank, and Stephen Coate. I also thank John Abowd, Marco Battaglini, Larry Blume, Ted O’Donoghue, Pradeep Dubey, Alex Imas, Jun Sung Kim, Sang-Hyun Kim, William D. Schulze, and Rasmus Wiese for their helpful comments. I gratefully acknowledge financial support from the Cornell Population Center and William D. Schulze. All the remaining errors are mine. 
environment to which they want to belong, based on some informative statistics about the environments. In the second phase, they choose their effort level, and after that their environmental support is realized. The second phase of the game can be interpreted in a manner similar to that of Lazear and Rosen (1981), namely, that the environmental support is the random or luck component. It is well known that in a many-player competition with a winner-take-all payoff structure, the variance of the environmental support (or of some random component in other contexts) can significantly affect the players' strategies and payoffs in equilibrium, and therefore it is straightforward to predict that the ranking of the expected environmental supports may not be consistent with the ranking of the expected utilities of the players.

My main research question was what ex-ante information is sufficient for choosing an optimal (i.e., expected payoff maximizing) environment, and whether economic agents will indeed choose the optimal environment. I was particularly interested in the situation where the best environment is not allowed to be chosen, so agents are asked to pick the second-best environment. The second-tier trap (STT) is a situation in which a player from the second-tier environment (the one with the second-largest expected environmental support) has the worst expected payoff even though his expected environmental support is strictly greater than that of the third-tier player. That is, when the best environment is unavailable and a sufficient condition for the STT is observed, players should choose the third-tier environment. I call this situation the STT because players are easily tempted to choose the second-tier environment over the second-best environment. The sufficient condition for the STT is so weak that even a sophisticated player could mistake the second-tier environment for the second-best one.

Though the model has the form of a competition among many identical players who exclusively choose one environment each, it would also serve as counterfactual analysis of an individual's irreversible life choices. We often encounter a situation where we must choose one of several exclusive options that will affect our life for a substantial period of time thereafter. Which college should I go to? Which major? Which career? Which social group? Even more challengingly, in several situations where someone else has chosen the most preferable option, or we are not accepted for that option, we have to choose one of the remaining options. The situation where the best choice is taken by someone else does not necessarily imply a difference in the abilities of those individuals (Frank, 2016). Identical $n$-player rank-order tournaments and Tullock contests theoretically predict the following: Every player exerts the same amount of effort, and one of them is randomly chosen to be the winner. In this case, we cannot say the 
winner is better than the others or that the winner exerts more effort. Indeed, as the number of competitors gets larger, it is more likely that the first-best option has already been taken by someone else, and so the other players have to choose the best option still available to them. This paper provides a novel approach to addressing this issue in the form of a two-stage competition: Just like all the life choices, a decision maker chooses an exclusive environment, and chooses an effort level to compete with other "counterfactual selves" who chose the road not taken. Since considering many selves, instead of many identical players, does not change the model and its predictions, all "identical players" in this paper may be thought of "counterfactual selves." However, for the sake of expositional simplicity, consistency with the laboratory experiment design, and potential relation to the existing literature, I maintain the interpretation of the model as a many-player competition.

In the first stage of my model, identical players reveal their preferences of environments and are assigned to environments according those preferences. If two or more players have the same preference, they are randomly assigned to different environments with equal probability. Alternatively, this could be thought of as a random ordering of identical players who take turns choosing the environment to which they want to belong. The player's environmental support is randomly drawn from an environment-specific support distribution at the end of the game. The support distribution can be interpreted as a different market situation that each player faces $;^{1}$ a characteristic of the group, such as a team, school, career, or social identity (Akerlof and Kranton, 2005); or simply a distribution of the luck component. Players know the support distributions, and are able to calculate some informative statistics, such as means, variances, and ex-ante advantages, at the time they are given a choice of environments. The ex-ante advantage of an environment is defined as the winning probability of a player from that environment when all the players exert the same amount of effort. In the second stage, players choose their level of effort, and environmental supports (accordingly, payoffs) are realized at the end of the game. The player with the highest output, which is the sum of the effort and the realization of the environmental support, wins the prize. Equilibrium payoffs are determined by the amount of the prize, the winning probability, and the cost of the effort.

\footnotetext{
${ }^{1}$ As an example of many-player competition, consider a situation where one of three workers will be promoted based on their outputs (the sum of their effort and the growth rate of the market), and the three workers will be in charge of separate international markets whose potential growth rates are random and heterogeneous. Before choosing their effort level, they have to exclusively choose one market whose growth rate is not yet realized. As an example of counterfactual analysis, consider a situation where a high school senior chooses a major for college. She tries to maximize her well-being (expected payoff minus cost of effort) but does not know which major will enable her to get a job that pays the most four years later.
} 
The tiers are defined according to the ranks of the expected environmental supports. Player $i$ is said to be in a higher tier than player $j$ if $E\left(\theta_{i}\right)>E\left(\theta_{j}\right)$. This definition is well justified, because equilibrium payoffs are consistent with tiers in pairwise competitions (Proposition 1). In three-player competitions, a sufficient condition for a player to attain a larger expected payoff by choosing the third-tier environment (the third-best environment in terms of the expected support) over the second-tier one is that the third-tier environment's ex-ante advantage be at least as large as that of the second-tier environment (Proposition 3). Since this is a sufficient condition, the second-best environment could be the third-tier environment even if the secondtier environment has a higher mean and a (slightly) higher ex-ante advantage than the third-tier one. I claim that players can easily be tempted to choose the second-tier environment when they are asked to choose the second-best environment, even though that would cause them to fall into the STT.

I conducted laboratory experiments to observe how subjects choose their environment to maximize their expected payoff. In each session, experiment participants played 10 rounds of rank-order tournament games. In the first stage of the game, each subject exclusively chose one of three environments, which were described by nonidentical uniform distributions. In the second stage, they chose an individual investment level on being given the attendant costs. At the end of the game, an environmental support was randomly drawn from the distribution, and payoffs were based on the rank of the outputs, where the output of each player was the sum of his environmental support and his individual investment. They chose one of three environments in four of the rounds (which I call the unrestricted rounds), and they were restricted to choose an environment other than the first-tier one in the other six rounds (the restricted rounds). None of the subjects chose the environment that maximized their expected payoffs in all the restricted rounds, while $52.57 \%$ of subjects chose the optimal environment in all the unrestricted rounds. On average, subjects choose the optimal environment in $28.67 \%$ of the restricted rounds and in $75.57 \%$ of the unrestricted rounds. Interestingly, those who always chose the optimal environment in the unrestricted rounds were more likely to fall into the STT. On average, they chose the optimal environment in only $16.67 \%$ of the restricted rounds. In the follow-up survey, most of the subjects indicated that they were more confident about their choices of environments than their choices of investment levels, and found that choosing the environment was an easier task than choosing an individual investment level.

The organization of the paper is as follows. In the following subsection I discuss the related 
literature. Section 2 presents the model, section 3 shows the relationship between the ranks of the expected environmental supports and the ranks of the equilibrium payoffs, and section 4 describes the experimental design and procedures. The experimental results are reported in section 5 , and section 6 concludes.

\subsection{Related Literature}

Following Lazear and Rosen (1981), many researchers have analyzed contests and all-pay auctions of asymmetric agents. This paper, from the model perspective, contributes to this literature. Some authors, including Krishna and Morgan (1998) and Moldovanu and Sela (2001), assume that ability includes some random components and that differences in ability are captured by the different and deterministic costs of effort. Nevertheless, they assume that the ability distributions are identical. Dubey (2013) takes stochastic ability and incomplete information into account. Pérez-Castrillo and Wettstein (2014) have a model in which abilities work in the same manner as environmental supports in this paper, but abilities are private information at the time that effort is made. The main differences between the existing literature and this paper are that all agents decide the level of effort before the random component is realized and the ability distributions are not identical across agents. To the best of my knowledge, there have been no models of all-pay auctions among three players that involve random environmental supports which are non-identical in terms of both means and variances. Therefore, the model presented here can be understood as a generalization of previous models. Once players are exclusively assigned to the environments in the first stage, the second stage of the model has a form similar to that of the Lazear-Rosen rank-order tournament model (Lazear and Rosen, 1981) and its descendants. Though rent-seeking behavior models are not the focus of my model, the equilibrium allocation of models presented in Nti (1999), Stein (2002), Allard (1988), and Tullock (1980) can be described as solutions of special cases of my model. ${ }^{2}$

Another difference between this paper and the existing literature is that there are more than two agents. When allowing for asymmetries among agents, most of the literature on allpay auction contests assumes that there are exactly two players as in Amann and Leininger (1996) or many ex-ante identical players as in Krishna and Morgan (1997). Some recent studies

\footnotetext{
${ }^{2}$ If the realizations of the environmental supports were publicly known before choosing the level of effort, then my model would reduce to the models presented in Nti (1999), Stein (2002), and Allard (1988). In this case, the ranks of the supports (abilities, or costs in some contexts) would yield a strong prediction of the ranks of the equilibrium payoffs. Moreover, if the environmental supports of all the players were non-stochastic and identical, then a player's probability of winning would be captured simply by the players' individual levels of effort relative to the aggregate sum of their efforts, which is a basic model provided by Tullock (1980).
} 
consider more than two agents. Siegel (2009) studies many-player all-pay contests to capture general asymmetries among contestants and analyzes the players' participation. Parreiras and Rubinchik (2010) consider three bidders in contests where participants have distinct risk preferences, and find that these differences can cause some players to drop out. Kirkegaard (2013) studies an incomplete information model among three groups where a "strong" group is handicapped, in order to allow for affirmative action, and shows that some of the players in the favored group may become worse off when the favored group is diverse. The thrust of this paper is similar to that of Kirkegaard (2013), since it shows that properties of two-bidder contests may not necessarily extend to many-bidder contests.

An analogue of this model is one where the environmental support distributions represent risks: The unrealized supports with the same mean can be represented by error terms with different variances. In this regard, this paper extends Hvide (2002), where two players with the same ability choose effort and risk levels; Kräkel and Sliwka (2004), where two players with different abilities choose effort and risk levels; and Gilpatric (2009), where three players in the contest choose one of the error terms, whose means are all 0 but whose variances vary. A fundamental difference between this paper and those studies is that the means of the support distributions are different and two players cannot choose the same distribution. When the support distributions represent only risks, and if players do not pay a penalty for low output, it is always optimal for them to choose a higher risk. This may not be the case when there is another support distribution which has a smaller variance but a higher mean. While the previous studies were about choices between effort and luck (risk), this study is more about choices between "higher mean and smaller variance" and "smaller mean and higher variance."

A large number of studies on competitions have used lab experiments (Bull, Schotter, and Weigelt, 1987; Schotter and Weigelt, 1992; Harbring and Irlenbusch, 2011; Agranov and Tergiman, 2013), including many studies reviewed above. ${ }^{3}$ However, the problem of exclusively choosing one of a finite set of heterogeneous supporting environments for a competition has not been considered, despite its importance. In many realistic situations, especially when making life choices, we first choose an environment which will support our effort, and those supporting environments do not necessarily have the same mean. Though the overall methodology of my laboratory experiments resembles that of Gneezy and Smorodinsky (2006), the goal of my experiments was to observe whether players can choose the environment that maximizes the

\footnotetext{
${ }^{3}$ For an extensive review of the experimental literature on contests, rank-order tournaments, and all-pay auctions, see Dechenaux, Kovenock, and Sheremeta (2014).
} 
expected payoff under complete information. ${ }^{4}$

\section{The Model}

Consider a two-stage game with $n \in\{2,3\}$ identical players indexed by $i \in\{1, \ldots, n\} \equiv N$, where the players compete for a prize. In the first stage, each player reveals his preference of $n$ heterogeneous environments (e.g., $n$ markets, $n$ schools, or $n$ careers) with a single-dimensional characteristic that randomly affects the outputs of the players. Throughout this paper, I call this characteristic an "environmental support" in that a player's choice of environment may provide benefits (or impose penalties) over and above those that stem from that player's effort. For example, every school may adopt different pedagogics. After students are disciplined in a particular academic environment, they may be randomly affected by the values emphasized by that school when they face a competitive job market. Let $G_{i}$ denote the continuous cumulative distribution for environment $i$ 's support, $\theta_{i}$. $\Theta_{i}$ denotes the support of $\theta_{i}$, where I assume that $\Theta_{i}=\mathbb{R}$ unless otherwise noted. Assume further that the probability density function for the support due to environment $i, g_{i}$, is unimodal and symmetric and has finite moments. With a slight abuse of terminology, I say that environment $i$ is where player $i$ belongs.

In the first stage, players know the support distributions and are able to calculate some informative statistics: means $\left(E\left(\theta_{i}\right)\right)$ and ex-ante advantages $\left(A_{i}\right) .{ }^{5}$ The ex-ante advantage of environment $i$ is defined as the winning probability of the player from environment $i$ when all the players exert the same amount of effort, that is,

$$
A_{i}=\int\left[\prod_{j \neq i} G_{j}(\theta)\right] g_{i}(\theta) d \theta, \quad i=1, \ldots, n .
$$

Since the same amount of effort neutralizes changes in the winning probabilities of all the players, the ex-ante advantages can be calculated by setting the effort made by every player to 0. I believe means and ex-ante advantages are, respectively, the first summary statistics when subjects look at each distribution separately and all distributions jointly. Once the players reveal their preferences, they are exclusively assigned to different environments. I assume the players

\footnotetext{
${ }^{4}$ The goal of my experiments is similar to that of Nieken (2010) in the sense that she allows subjects in two-player competitions to choose a variance for noise distributions, and finds that subjects did not choose the distribution with the higher variance, that is, the optimal distribution.

${ }^{5}$ If all the distributions are known and the players are rational, they will be able to calculate all the existing statistics which summarize the distributions. I specify their ability to calculate the means and ex-ante advantages, because I am considering the possibility of extending this model to one with bounded rationality.
} 
are identical, so all rational players will have the same preference order. Since identical players have identical preferences for environments, they are assigned randomly with equal probability. ${ }^{6}$ Alternatively, this setup could be thought of as a random ordering of identical players who take turns choosing the environment to which they want to belong.

In the second stage, players choose an effort level $e_{i} \geq 0$. The winner will receive a finite prize $w>0$, and the others will receive nothing. The probability of winning is determined by a player's output, $e_{i}+\hat{\theta}_{i}$, the sum of the effort level and the realization of the support due to environment $i$. Player $i$ 's effort costs him $c\left(e_{i}\right)$, where $c(\cdot)$ is a strictly increasing, twice continuously differentiable convex function. Player $i$ 's reward, $R_{i}$, is determined as follows:

$$
R_{i}\left(e_{i} \mid \hat{\theta}_{i}, \hat{\theta}_{-i}, e_{-i}\right)= \begin{cases}w & \text { if } e_{i}+\hat{\theta}_{i}=\max _{j \in N}\left\{e_{j}+\hat{\theta}_{j}\right\} \\ 0 & \text { otherwise }\end{cases}
$$

where the subscript $-i$ refers to all the players except $i .^{7}$ Each player's objective is to maximize

$$
E U_{i}\left(e_{i} \mid \theta_{i}, \theta_{-i}, e_{-i}\right) \equiv E\left[R_{i}\left(e_{i} \mid \theta_{i}, \theta_{-i}, e_{-i}\right)\right]-c\left(e_{i}\right)
$$

Note that exertion of a greater amount of effort will increase the probability of winning but will also increase the cost of the effort. After all players choose their level of effort, their payoffs are determined. Figure 1 summarizes the timing of events.

$\overbrace{\begin{array}{c}\text { Players reveal } \\ \text { preferences of } \\ \text { environments. }\end{array}}^{\begin{array}{c}\text { Players are } \\ \text { assigned to } \\ \text { environments. }\end{array}} \overbrace{\begin{array}{c}\text { Players } \\ \text { choose level } \\ \text { of effort. }\end{array}}^{\text {the first stage }} \quad \begin{gathered}\begin{array}{c}\text { Abilities and } \\ \text { payoffs are } \\ \text { realized. }\end{array} \\ \text { the second stage }\end{gathered}$

Figure 1: Sequence of Events in the Two-Stage Game

My first goal was to find a non-cooperative equilibrium for the second stage of the game. $G_{j}\left(\theta+e_{i}-e_{j}\right)$ is the probability that player $j$ 's realization of environmental support plus $e_{j}$ is

\footnotetext{
${ }^{6}$ This first stage is fairly simple, but it could allow for the possibility of adopting more general environments and preference matching algorithms in the manner of Gale and Shapley (1962). Though the model can be directly interpreted as one in which the number of environments is equal to the number of agents, I do not mean to impose such a severe restriction. Rather, I consider a situation where all other candidate-environment pairs have cleared the market and each of the three remaining candidates looks for an environment that has one spot left.

${ }^{7} \mathrm{~A}$ tie-break rule is unnecessary, because a tie will almost surely not occur.
} 
less than player $i$ 's output. Then the best response for player $i, B R_{i}\left(e_{-i}\right)$, will solve

$$
B R_{i}\left(e_{-i}\right) \in \arg \max _{e_{i} \geq 0} w \int\left[\prod_{j \neq i} G_{j}\left(\hat{\theta}+e_{i}-e_{j}\right)\right] g_{i}(\hat{\theta}) d \hat{\theta}-c\left(e_{i}\right),
$$

where $\int\left[\prod_{j \neq i} G_{j}\left(\hat{\theta}+e_{i}-e_{j}\right)\right] g_{i}(\hat{\theta}) d \hat{\theta} \equiv P_{i}\left(e_{i} \mid e_{-i}\right)$ is the probability that player $i$ wins when the effort choice is $e_{i}$, given $G_{-i}$ and $e_{-i}$. Note that when the realization of support is $\hat{\theta}$, player $i$ 's winning probability is equal to the product of the probabilities that all the other players' realizations of support $\hat{\theta}_{-i}$ given $e_{-i}$ are less than player $i$ 's realization of output. Since $G_{j}(\cdot)$ is a nondecreasing function whose lower limit is 0 and upper limit is $1, P_{i}\left(e_{i} \mid e_{-i}\right)$ also has these properties: $P_{i}\left(e_{i} \mid e_{-i}\right)$ is nondecreasing in $e_{i}, P_{i}\left(0 \mid e_{-i}\right) \geq 0\left(>0\right.$ when $G_{j}(x)>0$ for all $\left.x\right)$, and $\lim _{e_{i} \rightarrow \infty} P_{i}\left(e_{i} \mid e_{-i}\right)=1$. The winning probability for player $i$ is nondecreasing in $e_{i}$ and bounded above by 1 .

Since support is random when players choose their level of effort, the effort choice $e_{i}$ acts as a location shift parameter that shifts the support distribution of player $i$ 's environment. The best response of player $i$ is therefore determined by the amount of the location shift, given the other players' support distributions and location shifts.

The optimal choices of environment and effort can be described as a subgame perfect Nash equilibrium. In the second stage, a Nash equilibrium is a profile of effort choices, $e^{*}=$ $\left(e_{1}^{*}, \ldots, e_{n}^{*}\right)$, such that $e_{i}^{*} \in B R_{i}\left(e_{-i}^{*}\right)$ for all $i$. Note that $\lim _{e_{j} \rightarrow \infty} B R_{i}\left(e_{-i}\right)=0$ for every $j \neq i$, because if any contestant $j$ chooses a sufficiently large level of effort, player $i$ will not be compensated for the cost of any positive amount of effort. ${ }^{8}$ By Berge's maximum theorem, $B R_{i}\left(e_{-i}\right)$ is upper-semi continuous. Since the effort choice is always nonnegative, a pure strategy Nash equilibrium always exists for this game. ${ }^{9}$ In the first stage, the players reveal their preferences of environments according to the order of the expected payoffs in equilibrium.

\section{Analysis}

In this section, I focus mainly on analysis of the second stage. Because of the assumption that all the players have identical and rational preferences of environments according to the expected payoffs, the first stage of the problem becomes trivial.

\footnotetext{
${ }^{8}$ Suppose, for example, that the supports for all the players are drawn from the same distribution. If player $i$ chooses $e_{i} \geq c^{-1}(w)$, then the best response for all the other players is 0 .

${ }^{9}$ See Appendix A for an existence proof.
} 


\subsection{A Two-Player Competition}

I rank the environments by the expected supports of the distributions to check whether the ranks of the expected supports predict those of the equilibrium payoffs. Player $i$ is said to be in a higher tier than player $j$ if $E\left(\theta_{i}\right)>E\left(\theta_{j}\right) .{ }^{10}$ This definition of tiers is well justified, because the tiers are a strong predictor of the ranks of the equilibrium payoffs and the ranks of the effort levels in a pairwise competition. In a two-stage game, if players know only the expectations of the supports in the first stage, revealing preferences based on those expectations is optimal.

Proposition 1. Suppose $n=2$ and $\left(e_{1}^{*}, e_{2}^{*}\right)$ is an equilibrium. Then $E\left(\theta_{1}\right)>E\left(\theta_{2}\right)$ if and only if $E U_{1}^{*}\left(e_{1}^{*} \mid \theta_{1}, \theta_{2}, e_{2}^{*}\right)>E U_{2}^{*}\left(e_{2}^{*} \mid \theta_{2}, \theta_{1}, e_{1}^{*}\right)$. If $E\left(\theta_{1}\right)>E\left(\theta_{2}\right), e_{1}^{*} \geq e_{2}^{*}$ in any equilibrium.

Proof. See Appendix A.

Proposition 1 is intuitive when we consider a shift of the location parameter, $\theta_{1} \stackrel{d}{=} \theta_{2}+x$, for a deterministic $x>0$, so that $E\left(\theta_{1}\right)-E\left(\theta_{2}\right)=x>0$. Then $E\left[R_{1}\left(e_{1}^{*} \mid \theta_{1}, \theta_{2}, e_{2}^{*}\right)\right]-c\left(e_{1}^{*}\right)>$ $E\left[R_{2}\left(e_{2}^{*} \mid \theta_{2}, \theta_{1}, e_{1}^{*}\right)\right]-c\left(e_{2}^{*}\right)$, because the first-tier player has already been given a positive amount of "effort," $x$, for free. Now if $E\left[R_{1}\left(e_{1}^{*} \mid \theta_{1}, \theta_{2}, e_{2}^{*}\right)\right]-c\left(e_{1}^{*}\right)>E\left[R_{2}\left(e_{2}^{*} \mid \theta_{2}, \theta_{1}, e_{1}^{*}\right)\right]-c\left(e_{2}^{*}\right)$, then it cannot be the case that $E\left(\theta_{2}\right) \geq E\left(\theta_{1}\right)$. For if $E\left(\theta_{2}\right) \geq E\left(\theta_{1}\right)$, then $\left(e_{1}^{*}, e_{2}^{*}\right)$ is not an equilibrium, because for player $2, e_{1}^{*}$ is affordable and player 2's expected payoff can be at least as large as that of player 1 by choosing $e_{1}^{*}$. One obvious equilibrium is at $e_{1}^{*}=e_{2}^{*},{ }^{11}$ but uniqueness of equilibrium is not guaranteed.

Proposition 1 has two implications. First, the second and higher moments of the support distributions are irrelevant in determining the ranks of the expected payoffs in a pairwise competition; what matters is only the expectations of the supports. Second, the binary relation $E\left(\theta_{i}\right)>$ $E\left(\theta_{i+1}\right)$ implies transitivity of the equilibrium payoffs: If $E U_{1}^{*}\left(e_{1}^{*} \mid \theta_{1}, \theta_{2}, e_{2}^{*}\right)>E U_{2}^{*}\left(e_{2}^{*} \mid \theta_{2}, \theta_{1}, e_{1}^{*}\right)$ and $E U_{2}^{*}\left(e_{2}^{*} \mid \theta_{2}, \theta_{3}, e_{3}^{*}\right)>E U_{3}^{*}\left(e_{3}^{*} \mid \theta_{3}, \theta_{2}, e_{2}^{*}\right)$, then $E U_{1}^{*}\left(e_{1}^{*} \mid \theta_{1}, \theta_{3}, e_{3}^{*}\right)>E U_{3}^{*}\left(e_{3}^{*} \mid \theta_{3}, \theta_{1}, e_{1}^{*}\right)$. Thus, the definition of tiers is justified because it reveals the ranks of the equilibrium payoffs in a pairwise competition directly. Note also that the higher-tier player will not exert less effort than the lower-tier player in any equilibrium.

\footnotetext{
${ }^{10}$ Note that the definition of tier considers neither the higher moments of the distributions nor the players' effort choices. In addition, the fact that player 1 is in a higher tier than player 2 does not necessarily imply that $\theta_{1}$ firstorder stochastically dominates $\theta_{2}$. For example, if $\theta_{1} \sim U[2,4]$ and $\theta_{2} \sim U[0,5]$, where $U[a, b]$ is a uniform distribution between $a$ and $b$, then player 1 is in a higher tier than player 2, even though the variance and the maximum possible support of the second tier are greater than those of the first tier.

${ }^{11}$ The first-order condition (FOC) for player $i$ is $\int g_{j}\left(\theta+e_{i}-e_{j}^{*}\right) g_{i}(\theta) d \theta-c^{\prime}\left(e_{i}\right)=0$. Similarly the FOC for player $j$ is $\int g_{i}\left(\theta+e_{j}-e_{i}^{*}\right) g_{j}(\theta) d \theta-c^{\prime}\left(e_{j}\right)=0$. When $e_{i}=e^{*}=e_{j}$, both FOCs are satisfied.
} 
Suppose now the ranks of the environments are determined by a different statistic of the environments, namely, their ex-ante advantages. The ex-ante advantage of environment $i, A_{i}$, is calculated by (1). For example, if $\theta_{1}=3$ and $\theta_{2} \sim U[0,4]$, then $A_{1}=3 / 4$ and $A_{2}=1 / 4$. In a two-player competition, the ranks of the ex-ante advantages are consistent with the ranks of the expected environmental supports, ${ }^{12}$ and thus, by Proposition 1, they are consistent with the equilibrium payoffs as well as with the equilibrium effort levels.

Since the means and the ex-ante advantages convey sufficient information about the ranks of the expected payoffs, the players can simply reveal preferences according to the tiers in the first stage. Whether they have complete information of the support distributions is irrelevant.

\subsection{A Three-Player Competition}

In a three-player competition, the ranks of the expected supports can be inconsistent with the ranks of the equilibrium payoffs and with the ranks of the ex-ante advantages. It is well known that the variances of the support distributions are a significant factor in the ranks of the equilibrium payoffs, so this claim is straightforward. At this point, I provide several numerical examples in which $c(e)$ is set to $e^{2}$, for two purposes: to observe some regularities and to illustrate setups similar to those used in the laboratory experiments. To minimize unnecessary complexity, all the examples have a unique equilibrium. Consider Example 1, shown in Table 1. If $\theta_{1}=5, \theta_{2} \sim U[3,6]$, and $\theta_{3} \sim U[0.5,7.5]$, then $E\left(\theta_{1}\right)>E\left(\theta_{2}\right)>E\left(\theta_{3}\right) .{ }^{13}$ However, the ex-ante advantage and the equilibrium payoff for player 2 are smaller than those for player 3.

\begin{tabular}{|c|c|c|c|c|}
\hline Example 1 & Player 1 & Player 2 & Player 3 & Rank \\
\hline$\theta_{i}$ & 5 & $U[3,6]$ & $U[0.5,7.5]$ & - \\
\hline$E\left(\theta_{i}\right)$ & 5 & 4.5 & 4 & $E\left(\theta_{1}\right)>E\left(\theta_{2}\right)>E\left(\theta_{3}\right)$ \\
\hline$A_{i}$ & 0.4286 & 0.2481 & 0.3333 & $\mathbf{A}_{1}>\mathbf{A}_{3}>\mathbf{A}_{2}$ \\
\hline$e_{i}^{*}($ when $w=1)$ & 0.2332 & 0.1324 & 0.0714 & $e_{1}^{*}>e_{2}^{*}>e_{3}^{*}$ \\
\hline$E U_{i}^{*}\left(e_{i}^{*} \mid \Theta, e_{-i}^{*}\right)$ & 0.4120 & 0.2013 & 0.3097 & $\mathbf{E U}_{\mathbf{1}}^{*}>\mathbf{E U}_{\mathbf{3}}^{*}>\mathbf{E} \mathbf{U}_{\mathbf{2}}^{*}$ \\
\hline
\end{tabular}

Table 1: Tiers $\not$ Ranks of the equilibrium payoffs. Tiers $\not$ Ranks of the ex-ante advantages.

The ranks of the ex-ante advantages also do not predict the ranks of the equilibrium payoffs. When $\theta_{1}=5, \theta_{2} \sim U[3,6]$, and $\theta_{3} \sim U[0.5,6.8]$, as shown in Table $2, A_{1}>A_{2}>A_{3}$, but still the STT arises. That is, the second-tier player is trapped despite obviously being better than the third-tier player in the pairwise competition, but is the worst in terms of the expected

\footnotetext{
${ }^{12}$ See Lemma 1 in Appendix A.

${ }^{13}$ I set $\theta_{1}$ to a constant for convenience. The main result is independent of this simplification.
} 
equilibrium payoff in a three-player competition, even though the second-tier player exerts more effort than the third-tier player.

\begin{tabular}{ccccc}
\hline Example 2 & Player 1 & Player 2 & Player 3 & Rank \\
\hline$\theta_{i}$ & 5 & $U[3,6]$ & $U[0.5,6.8]$ & - \\
\hline$E\left(\theta_{i}\right)$ & 5 & 4.5 & 3.65 & $E\left(\theta_{1}\right)>E\left(\theta_{2}\right)>E\left(\theta_{3}\right)$ \\
\hline$A_{i}$ & 0.4762 & 0.2646 & 0.2593 & $A_{1}>A_{2}>A_{3}$ \\
\hline$e_{i}^{*}($ when $w=1)$ & 0.2620 & 0.1473 & 0.0794 & $e_{1}^{*}>e_{2}^{*}>e_{3}^{*}$ \\
\hline$E U_{i}^{*}\left(e_{i}^{*} \mid \Theta, e_{-i}^{*}\right)$ & 0.4553 & 0.2184 & 0.2297 & $\mathbf{E U}_{\mathbf{1}}^{*}>\mathbf{E U _ { 3 } ^ { * }}>\mathbf{E U}_{\mathbf{2}}^{*}$ \\
\hline
\end{tabular}

Table 2: Ranks of the ex-ante advantages $\not$ Ranks of the equilibrium payoffs.

Greater effort on the part of the second-tier player does not necessarily cause him to have a lower equilibrium payoff. The second-tier player always has a higher equilibrium payoff with a higher level of effort in the pairwise competition. This is also true in many cases in a threeplayer competition, as shown in the example in Table 3. In addition, the ranks of the equilibrium effort choices cannot be predicted by the tiers or by the ex-ante advantages. To summarize, unlike a two-player competition, the tiers can be inconsistent with the ex-ante advantages, and neither the tiers nor the ex-ante advantages reveal the order of the equilibrium payoffs or of the equilibrium effort levels.

\begin{tabular}{ccccc}
\hline Example 3 & Player 1 & Player 2 & Player 3 & Rank \\
\hline$\theta_{i}$ & 5 & $U[3,6]$ & $U[1.6,6.4]$ & - \\
\hline$E\left(\theta_{i}\right)$ & 5 & 4.5 & 4 & $E\left(\theta_{1}\right)>E\left(\theta_{2}\right)>E\left(\theta_{3}\right)$ \\
\hline$A_{i}$ & 0.4722 & 0.2708 & 0.2569 & $A_{1}>A_{2}>A_{3}$ \\
\hline$e_{i}^{*}($ when $w=1)$ & 0.2599 & 0.1545 & 0.1042 & $e_{1}^{*}>e_{2}^{*}>e_{3}^{*}$ \\
\hline$E U_{i}^{*}\left(e_{i}^{*} \mid \Theta, e_{-i}^{*}\right)$ & 0.4523 & 0.2248 & 0.2206 & $E U_{1}^{*}>E U_{2}^{*}>E U_{3}^{*}$ \\
\hline
\end{tabular}

Table 3: Greater effort of the second-tier player does not cause a lower equilibrium payoff.

One useful observation can be obtained by a comparison of Examples 1 and 3, which is summarized in Table 4. Note that $U[0.5,7.5]$ is a mean-preserving spread of $U[1.6,6.4]$. If the variance of the third-tier environment gets larger, the ex-ante advantage of this environment will also get larger, while the ex-ante advantages of the other environments will get smaller. This is because the third-tier player has more of a possibility for a higher realization of support. ${ }^{14}$ Every player's equilibrium effort choice will decrease in such a scenario, because the increased uncertainty in the environmental support will dilute the marginal benefit of the effort. Proposition 2 states how a mean-preserving spread affects equilibrium payoffs.

\footnotetext{
${ }^{14}$ Of course, he also has more of a possibility for a lower realization of support, but under the winner-take-all payoff structure, such possibility does not affect the ex-ante advantage.
} 


\begin{tabular}{|c|c|c|c|c|}
\hline Example 1 and 3 & $E\left(\theta_{i}\right)$ & $A_{i} \Rightarrow A_{i}^{\prime}$ & $e_{i}^{*} \Rightarrow e_{i}^{* \prime}$ & $E U_{i}^{*} \Rightarrow E U_{i}^{* \prime}$ \\
\hline$\theta_{1}=5$ & 5.0 & $0.4772 \Rightarrow 0.4286(\Downarrow)$ & $0.2599 \Rightarrow 0.2332(\Downarrow)$ & $0.4523 \Rightarrow 0.4120(\Downarrow)$ \\
\hline$\theta_{2} \sim U[3,6]$ & 4.5 & $0.2708 \Rightarrow 0.2481(\Downarrow)$ & $0.1545 \Rightarrow 0.1324(\Downarrow)$ & $0.2248 \Rightarrow 0.2013(\Downarrow)$ \\
\hline $\begin{aligned} \theta_{3} & \sim U[1.6,6.4] \\
\Rightarrow \theta_{3}^{\prime} & \sim U[0.5,7.5]\end{aligned}$ & 4.0 & $0.2569 \Rightarrow 0.3333(\Uparrow)$ & $0.1042 \Rightarrow 0.0714(\Downarrow)$ & $0.2206 \Rightarrow 0.3097(\Uparrow)$ \\
\hline
\end{tabular}

Table 4: Changes Due to a Mean-Preserving Spread of $\theta_{3}$

Proposition 2. Suppose $n=3$ and $E\left(\theta_{1}\right)>E\left(\theta_{2}\right)>E\left(\theta_{3}\right)$. A mean-preserving spread of $\theta_{i}, i=2,3$, causes $A_{i}$ to increase, $A_{-i}$ to decrease, and $e^{*}=\left(e_{1}^{*}, e_{2}^{*}, e_{3}^{*}\right)$ to decrease. Thus $E U_{i}^{*}\left(e_{i}^{*} \mid \theta_{1}, \theta_{2}, \theta_{3}, e_{-i}^{*}\right)$ increases, but $E U_{j}^{*}\left(e_{j}^{*} \mid \theta_{1}, \theta_{2}, \theta_{3}, e_{-j}^{*}\right)$ does not increase for any $j \neq i$.

Proof. See Appendix A.

Proposition 2 implies that the ranks of the equilibrium payoffs can be changed as a result of a change in the variance of the support of a lower-tier environment. In other words, if the variance of the support of a lower-tier environment does not exceed that of a higher-tier one, the STT does not arise. $\operatorname{Var}\left(\theta_{3}\right)>\operatorname{Var}\left(\theta_{2}\right)$ is a necessary condition for the STT.

Corollary 1. [A necessary condition for the second-tier trap] Consider a positive number $x$ and assume $\tilde{\theta}_{3} \stackrel{d}{=} \theta_{2}-x$, so that $\operatorname{Var}\left(\theta_{2}\right)=\operatorname{Var}\left(\tilde{\theta}_{3}\right)$. Then $\operatorname{EU}_{2}^{*}\left(e_{2}^{*} \mid \theta_{1}, \theta_{2}, \tilde{\theta}_{3}, e_{-2}^{*}\right) \geq$ $E U_{3}^{*}\left(e_{3}^{*} \mid \theta_{1}, \theta_{2}, \tilde{\theta}_{3}, e_{-3}^{*}\right)$. There exists a mean-preserving spread of $\tilde{\theta}_{3}$, denoted by $\theta_{3}$, such that $E U_{3}^{*}\left(e_{3}^{*} \mid \theta_{1}, \theta_{2}, \theta_{3}, e_{-3}^{*}\right)>E U_{2}^{*}\left(e_{2}^{*} \mid \theta_{1}, \theta_{2}, \theta_{3}, e_{-2}^{*}\right)$ and $\operatorname{Var}\left(\theta_{3}\right)>\operatorname{Var}\left(\tilde{\theta}_{3}\right)=\operatorname{Var}\left(\theta_{2}\right)$.

Proof. See Appendix A.

To understand this necessary condition, consider the case where $\theta_{1} \stackrel{d}{=} \theta_{3}+x$ and $\theta_{2} \stackrel{d}{=} \theta_{3}+y$ for positive numbers $x$ and $y$ with $x>y$. Then the necessary condition for the STT is not satisfied, because the $\theta_{i}$ 's have the same variance. In this case, the first-tier (resp. second-tier) player will always be better off by keeping the level of effort the same as that of the second-tier (resp. third-tier) player, because the cost of effort will be the same but the higher-tier player has the greater chance of winning. With the same level of effort, the rank of the expected payoffs is mainly determined by the ex-ante advantages, which are always $A_{1}>A_{2}>A_{3}$ in this case. Thus there is no STT.

The following proposition states a sufficient condition for the existence of the STT.

Proposition 3. [A sufficient condition for the second-tier trap] In a three-player competition, if $E\left(\theta_{1}\right)>E\left(\theta_{2}\right)>E\left(\theta_{3}\right)$ and $A_{2}=A_{3}$, then $E U_{3}^{*}\left(e_{3}^{*} \mid \theta_{1}, \theta_{2}, \theta_{3}, e_{-3}^{*}\right) \geq E U_{2}^{*}\left(e_{2}^{*} \mid \theta_{1}, \theta_{2}, \theta_{3}, e_{-2}^{*}\right)$. 
Proof: See Appendix A.

When combined with Proposition 2, this sufficient condition implies that the STT can also arise if $A_{3}>A_{2}$. This sufficient condition for the STT can be interpreted in terms of the source of the ex-ante advantages. $A_{2}=A_{3}$ and $E\left(\theta_{2}\right)>E\left(\theta_{3}\right)$ jointly imply that the second-tier player's ex-ante advantage is driven by the first moment of the support distribution, while the third-tier player's ex-ante advantage is driven by the second moment. Intuitively speaking, when the second-tier environment is not as supportive as the first-tier environment in terms of the mean, and is not as advantageous as the third-tier environment, it is wise to conclude that the second-tier environment is not the second-best one. The sufficient condition also implies that the two statistics, means and ex-ante advantages, are "almost" enough to determine the second-best environment. The STT can arise even when the second-tier environment has a higher mean and a (slightly) higher ex-ante advantage than the third-tier environment, as illustrated in Example 2 .

\subsection{The First Stage}

Since the second stage has been analyzed, dealing with the first stage is straightforward. Indeed, the distinction between the first and the second stages is not theoretically meaningful, because identical players will have the same preferences for any set of different environments. I frame the model as a two-stage game so that the model will be a better fit with the experimental design.

If all the players are rational, then they will prefer the third-tier environment over the secondtier one in cases where the ex-ante advantages of the second- and the third-tier environments are similar. Therefore, when a subject is told that one environment is already taken by someone else and she has to choose from the other available environments, she should choose the second-best environment. However, I claim that the sufficient condition is less intuitive for naïve decision makers, and hence that the players can be tempted to choose the second-tier environment over the second-best one when those are not the same. In particular, if subjects follow their own rule of thumb, for example, always choose the highest available expected support or the highest available ex-ante advantage, then they may place themselves in the worst environment even though they have several options to choose from. One experimentally interesting question is 
how they decide the investment level (the effort choice in the theoretical framework) when they choose a "wrong" environment.

\section{Experimental Design and Procedures}

The lab experiments were designed to observe how subjects choose their environment to maximize their expected payoffs. The experiments were conducted at the Cornell Lab for Experimental Economics \& Decision Research and employed undergraduate students at Cornell University for ten sessions and graduate students in math-related fields for two sessions. ${ }^{15} \mathrm{~A}$ total of 175 students (154 undergraduates and 21 graduates) participated in an experimental session, and the average payment, including a reward for participation, was $\$ 12.61$.

A general description of the experiments is as follows: Each session consisted of ten rounds of winner-take-all competitions in which the setup was similar to the model presented in section 2. During the experiments, they earned "points," which were converted to U.S. dollars at the end of the experiments at the rate of 1,000 points $=1$ USD. In each round, subjects were endowed with 40 virtual "tokens" and competed with two players (both of which were computers). In the first phase of each round, they exclusively chose one of several environments, which were described by uniform distributions, and then in the second phase they chose their investment level in terms of tokens. At the end of every round, the environmental support for each player was drawn randomly from the distribution for that player's chosen environment. The winner was the one whose output (= individual investment + environmental support) was largest. The net payoff per round was $1600-X^{2}+10(40-X)$ when the subject won, and $-X^{2}+10(40-X)$ when s/he lost, where $X \in[0,40]$ was the amount of the token investment. In theory, the reward per round could vary from 0 points (when the subject invested 40 tokens and lost) to 1600 points or 1.6 dollars (when the subject invested 0 tokens and won). The earned points were accumulated to their account. Subjects were endowed with 7,000 points (the show-up fee) at the beginning of the experiment, so that subjects would never be constrained in their investment choices on account of insufficient points in the course of the experiment.

Table 5 summarizes the construction of the experiments. In four of the rounds $(2,4,7$, and 9), subjects chose one of three environments. I call these the unrestricted rounds. In the other six

\footnotetext{
${ }^{15}$ In recruiting graduate students, I added the following sentence to the eligibility description: "The study is open to Cornell graduate students who can calculate mathematical expectations." I also asked them to fill in their major on the sign-in form, and all of those who participated were in math-related fields, such as computer science, economics, operations research, and electrical engineering. The purpose of inviting graduate students was to check whether the mathematical understanding of the subjects affects the experimental observations.
} 
rounds, which I call the restricted rounds, they were informed that one environment (the first-tier environment ${ }^{16}$ ) had already been taken by a competitor, and they were to choose one of the two remaining environments. I was able to identify their preferences of environments by interpreting their selection in the unrestricted rounds to be their preferred environment and their selection in the restricted rounds to be their second choice. (I assumed that their first choice in the restricted rounds would have been the first-tier environment.) To exclude the possibility of choices made as a result of miscalculation, the mean of each support distribution was displayed. I also provided a simulator that could be used to calculate the winning probabilities for any contingent set of effort choices (see Figure 2). Without any additional action on their part, subjects were able to obtain averages and ex-ante advantages, which were sufficient to determine the environment that maximized the expected equilibrium payoff in the restricted rounds. A simple calculator and scratch paper were also provided, and I encouraged subjects to use them whenever necessary. I acknowledge that providing averages and ex-ante advantages may have been interpreted as particular rules of thumb and that subjects may simply have used the information provided as their guide. I also realize that subjects may have found that using the simulator to calculate expected payoffs was a complicated operation. To address these issues, I also ran supplementary experiments: one in which 39 subjects were provided with a different simulator - one that could be used to directly calculate the expected payoffs for any contingent set of effort choices - and one in which 40 subjects were provided with no simulator and not even the means of the support distributions were displayed (see Figure 3). Note that when the expected payoff calculator was provided, subjects would have been able to find a Nash equilibrium by carrying out three or four operations with the simulators. In neither the restricted rounds nor the unrestricted rounds did I find any significant differences between results from the main experiment and those from the supplementary experiments, so I pooled them. ${ }^{17}$

The support distributions varied from one round to another, but were specified in such a way that the STT arose in every round, that is, if not given the option of choosing the first-tier environment, a fully rational agent would choose the third-tier environment even though the second-tier one may seem more attractive. In rounds 1,4 , and 8 , the second-tier environment's ex-ante advantage was slightly greater than that of the third-tier one. In rounds 2 and 7 , the ex-

\footnotetext{
${ }^{16}$ I deliberately did not use terms that could imply anything about ranks or orders. Subjects were simply told that "one of the environments" had already been taken.

${ }^{17}$ Under the null hypothesis that environment choices in the restricted rounds observed from the main experiment and those from the supplementary experiments come from the same distribution, the $z$-value from the Mann-Whitney $U$ test was $0.7386\left(n_{1}=576, n_{2}=474, U=140124\right.$, two-tailed $)$. In the unrestricted rounds, the difference was a little larger but not statistically significant ( $z$-value: $1.8516, n_{1}=384, n_{2}=316, U=138170$, two-tailed).
} 


\begin{tabular}{cccccccc}
\hline Round & Env. A & Env. B & \multirow{2}{*}{ Env. C } & $\begin{array}{c}\text { Available } \\
\text { options }\end{array}$ & $\begin{array}{c}\text { Optimal } \\
\text { choice }\end{array}$ & $\begin{array}{c}\text { Ranks of } \\
\text { the means }\end{array}$ & $\begin{array}{c}\text { Ranks of the exp. } \\
\text { eqm. payoffs }\end{array}$ \\
\hline 1 & {$[3.80,68.87]$} & {$[27.28,58.73]$} & {$[18.84,62.38]$} & $A, C$ & $A$ & $B>C>A$ & $B>A>C$ \\
\hline 2 & {$[23.03,65.87]$} & {$[6.49,73.06]$} & {$[29.97,63.42]$} & $A, B, C$ & $C$ & $C>A>B$ & $C>B>A$ \\
\hline 3 & {$[33.92,78.13]$} & {$[25.48,80.78]$} & {$[10.44,88.77]$} & $B, C$ & $C$ & $A>B>C$ & $A>C>B$ \\
\hline 4 & {$[33.50,77.04]$} & {$[41.94,73.39]$} & {$[18.46,83.53]$} & $A, B, C$ & $B$ & $B>A>C$ & $B>C>A$ \\
\hline 5 & {$[1.52,79.85]$} & {$[16.56,71.86]$} & {$[25.00,69.21]$} & $A, B$ & $A$ & $C>B>A$ & $C>A>B$ \\
\hline 6 & {$[33.17,110.47]$} & {$[50.61,105.82]$} & {$[18.13,117.76]$} & $A, C$ & $C$ & $B>A>C$ & $B>C>A$ \\
\hline 7 & {$[35.86,69.31]$} & {$[12.38,78.95]$} & {$[28.92,71.76]$} & $A, B, C$ & $A$ & $A>C>B$ & $A>B>C$ \\
\hline 8 & {$[32.78,64.23]$} & {$[9.30,74.37]$} & {$[24.34,67.88]$} & $B, C$ & $B$ & $A>C>B$ & $A>B>C$ \\
\hline 9 & {$[31.64,86.94]$} & {$[16.60,94.93]$} & {$[40.08,84.29]$} & $A, B, C$ & $C$ & $C>A>B$ & $C>B>A$ \\
\hline 10 & {$[20.82,120.45]$} & {$[35.86,113.16]$} & {$[53.30,108.51]$} & $A, B$ & $A$ & $C>B>A$ & $C>A>B$ \\
\hline
\end{tabular}

Table 5: Construction of Experiments

The second through fourth columns show the support distributions. For example, if a participant chose environment A in round 1, the environmental support was randomly drawn from the uniform distribution between 3.80 and 68.87 . The fifth column shows the available options. When only two options were available, subjects were told that the other one had been chosen by one of the computer players. The sixth column shows the choice of environment that maximized the expected equilibrium payoff given the available options. The seventh column shows the ranks of the expectations, and the last column shows the ranks of the expected equilibrium payoffs.

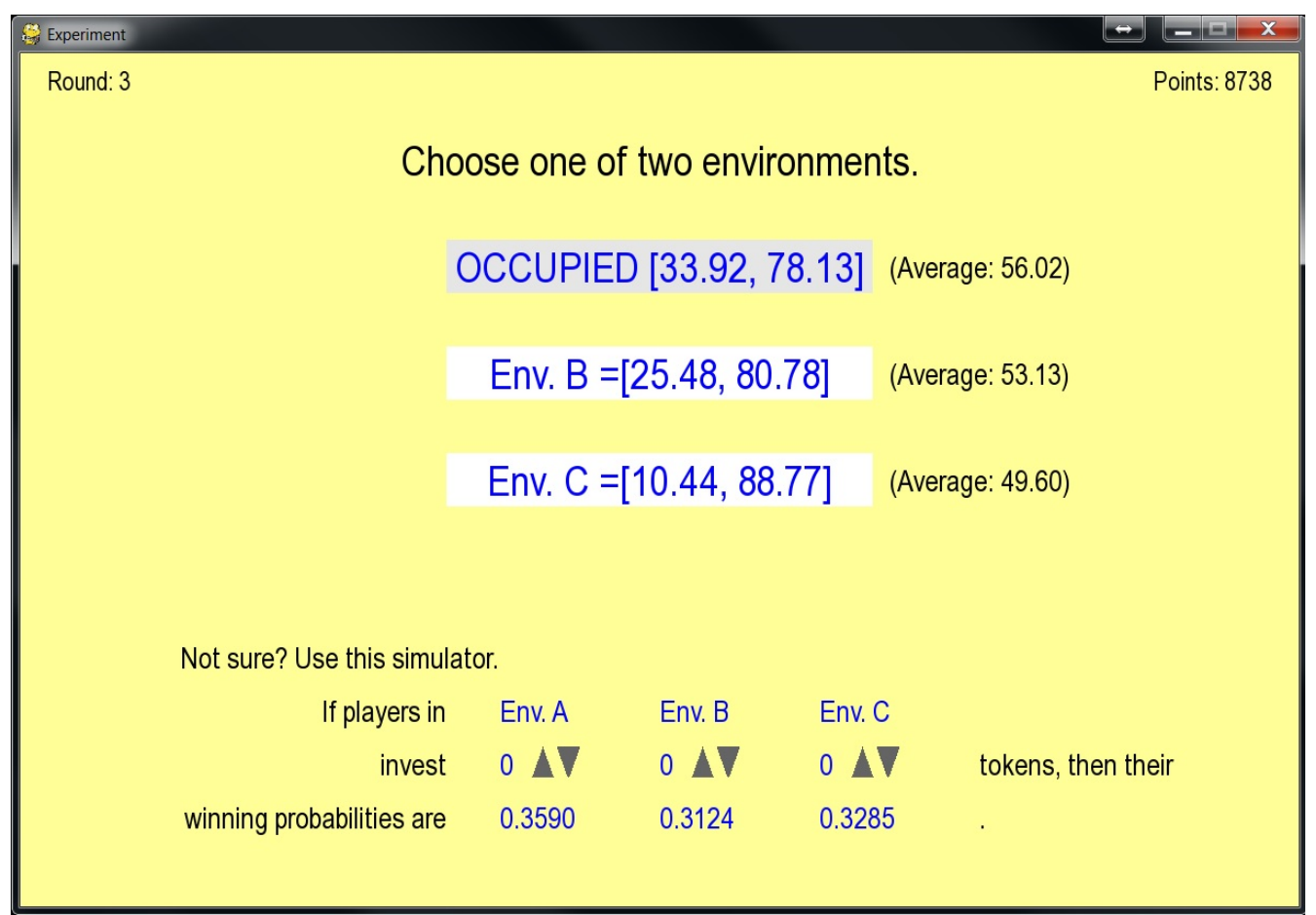

Figure 2: Screen Capture of the First Stage of Round 3 in the Main Experiment Subjects were informed that environment A had already been chosen by a competitor, so they chose either environment $\mathrm{B}$ or environment C. Without any further action or calculation, they obtained the means and ex-ante advantages of the support distributions. (The ex-ante advantages are the probabilities of winning when all the players invest 0 .) Since the mean of environment B is greater than that of environment $\mathrm{C}$ and the ex-ante advantage of environment $\mathrm{C}$ is greater than that of environment $\mathrm{B}$, they should have chosen environment $\mathrm{C}$ to maximize their expected payoff. 


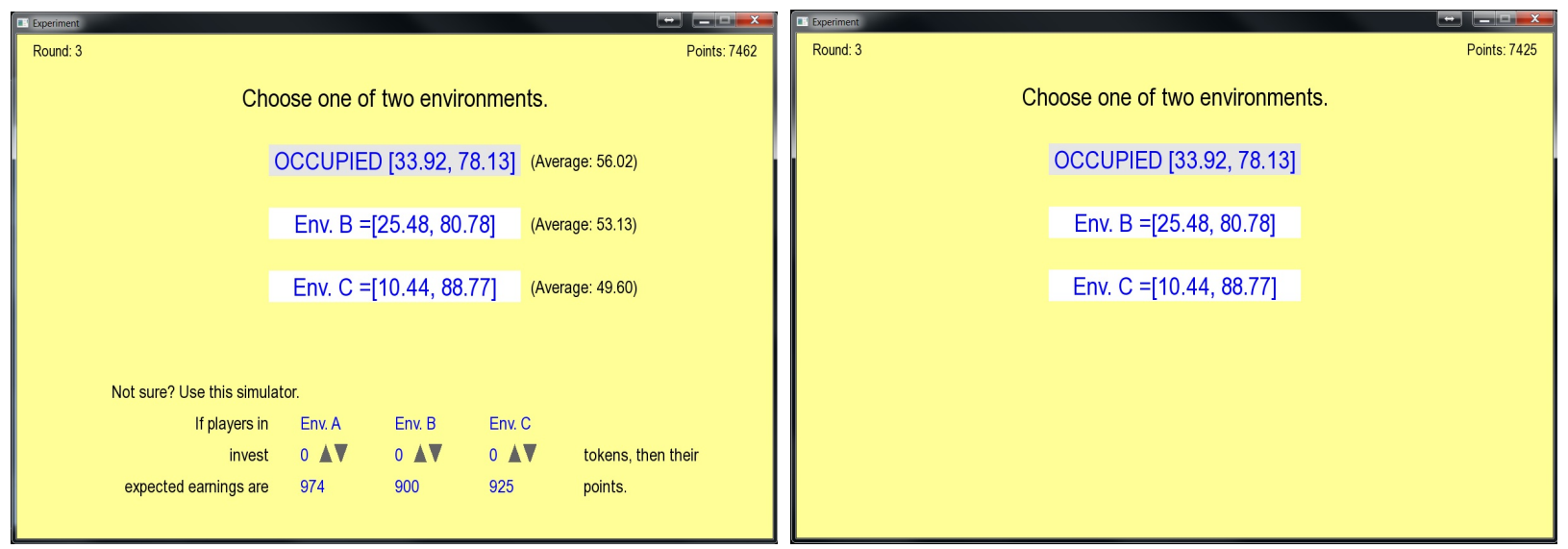

Figure 3: Screen Captures of the First Stage of Round 3 in the Supplementary Experiments Left: A simulator that could be used to calculate the expected payoffs for any contingent set of effort choices. Subjects could find a Nash equilibrium by carrying out a few operations with this simulator. Right: Any information that could have been used as a guide was not provided.

ante advantages were almost the same. In rounds $3,5,6,9$, and 10, the third-tier environment's ex-ante advantage was slightly greater than that of the second-tier one. The environments were displayed in random order, to prevent subjects from inferring the advantages of the environments from their order on the display.

Subjects were also told that the two computer competitors would make their own optimal investment decisions, and would assume that subjects would choose their best investment decisions. ${ }^{18}$ Since subjects knew that the computer players' investment choices were independent of their choices, they did not worry about contingent decision changes of the other players. At the end of every round, environmental supports were drawn from the respective distributions, and subjects were informed whether they won and how much they earned (or lost). The computer players' investment choices were not divulged, because they always chose the same action when given the same conditions.

Before participating in the experiments, subjects followed the instructions on the tutorial screen and answered six multiple choice questions to check their understanding of the instructions. They were allowed to participate in the experiments only if all of their answers were correct. See Appendix B for the screening test questions. 23 undergraduates failed to pass the screening test. After passing the screening test, subjects played two practice rounds (one

\footnotetext{
${ }^{18}$ Some graduate participants may have recognized that I preset the two computer competitors to always choose Nash equilibrium effort levels, but I did not want to make the task too complex for the subjects. I provided a more detailed explanation whenever subjects did not fully understand the instructions. I focused on conveying two messages: (1) Once a subject choses an environment, the other (computer) players were exclusively assigned to the other environments. (2) The computer players' unobserved investment levels were preset, so they would not change their investment decisions no matter what the subject chose as his/her investment level.
} 
unrestricted and one restricted) without payment.

After the experiments, subjects were surveyed on their confidence in their decisions. This follow-up survey consisted of the following two sets of two questions:

1.1. "Overall, which of the following is the closest description of your choices of environments?" (followed by four sentences describing different levels of confidence)

1.2. "Overall, which of the following is the closest description of your choices of investments?" (followed by four sentences describing different levels of confidence)

2.1. "Overall, how hard was it to choose an environment?" (followed by a five-level Likert scale, from "very easy" to "very hard")

2.2. "Overall, how hard was it to choose an investment level?" (followed by a five-level Likert scale, from "very easy" to "very hard")

\section{Results}

The main results from the lab experiments can be summarized as follows:

1. Subjects chose the optimal environment in $75.57 \%$ of the unrestricted rounds (529 out of 700 rounds). However, they chose the optimal environment in only $28.67 \%$ of the restricted rounds (301 out of 1050 rounds). That is, subjects were likely to fall into the STT.

2. The 92 subjects who chose the optimal environment in all the unrestricted rounds were more likely to fall into the STT. No one chose the optimal environment in all six restricted rounds. In fact, those 92 subjects chose the optimal environment in only $16.67 \%$ of the restricted rounds.

3. Those 92 subjects were more confident in their choices of environments, though they were less likely than their counterparts to choose the optimal environment. They also reported that choosing environments was an easier task than choosing investment levels.

Those observations strongly suggest that the STT consistently arose. The level of understanding of optimization problems did not help them to do better. There was no significant difference between sessions with undergraduate students and those with graduate students. ${ }^{19}$ In fact, the average payment for the graduate student sessions was slightly lower than that of the undergraduate student sessions, and a slightly smaller proportion of graduate students chose

\footnotetext{
${ }^{19}$ Under a null hypothesis of the same proportion of the correct environment choices, the $t$-statistic of the proportion difference of the two subgroups is 0.4335 .
} 
the optimal environment, though differences in choice patterns between undergraduate and graduate subjects were not significant. ${ }^{20}$ Also there was no noticeable difference by gender. ${ }^{21}$

\begin{tabular}{ccccccccccc}
\hline Round & 1 & 2 & 3 & 4 & 5 & 6 & 7 & 8 & 9 & 10 \\
\hline Game Type & $\mathrm{R}$ & $\mathrm{U}$ & $\mathrm{R}$ & $\mathrm{U}$ & $\mathrm{R}$ & $\mathrm{R}$ & $\mathrm{U}$ & $\mathrm{R}$ & $\mathrm{U}$ & $\mathrm{R}$ \\
SPNE & $(\mathrm{A}, 7)$ & $(\mathrm{C}, 16)$ & $(\mathrm{C}, 5)$ & $(\mathrm{B}, 17)$ & $(\mathrm{A}, 5)$ & $(\mathrm{C}, 3)$ & $(\mathrm{A}, 16)$ & $(\mathrm{B}, 7)$ & $(\mathrm{C}, 12)$ & $(\mathrm{A}, 3)$ \\
Mode (env. choice) & $\mathrm{C}$ & $\mathrm{C}$ & $\mathrm{B}$ & $\mathrm{B}$ & $\mathrm{B}$ & $\mathrm{A}$ & $\mathrm{A}$ & $\mathrm{C}$ & $\mathrm{C}$ & $\mathrm{B}$ \\
Correct decision (\%) & 20.00 & 74.86 & 33.14 & 78.86 & 34.29 & 32.00 & 73.14 & 20.00 & 75.43 & 32.57 \\
\hline Avg. inv. choice & 13.73 & 16.19 & 15.42 & 16.69 & 14.19 & 14.51 & 16.21 & 14.41 & 16.34 & 14.55 \\
Overinvestment & 5.10 & 5.66 & 8.96 & 5.13 & 7.83 & 10.67 & 5.84 & 5.72 & 8.39 & 10.35 \\
\hline
\end{tabular}

Table 6: Summary: All Subjects

This table shows how well subjects did in finding the environment that maximized their expected payoff in equilibrium. In the second row, $R$ stands for a restricted round and $U$ stands for an unrestricted round. The third row shows the subgame perfect Nash equilibrium strategy for the given constraints, where the first entity is the optimal environment choice and the second one is the optimal investment choice. The fourth row shows the most frequently chosen environment. The fifth row shows the proportion of subjects who chose the optimal environment. The sixth row shows the average of the individual investment choices. In the last row, overinvestment is the mean difference between the optimal investment choices for the players' chosen environments and their actual investment choices.

Table 6 shows some basic descriptive statistics about subjects' environment choices by round.

The fifth row of the table shows the proportion of subjects who chose the optimal environment.

For the unrestricted rounds, denoted by $\mathrm{U}$ in the table, their choices were quite good overall:

In each unrestricted round, $73.14 \%$ or more of subjects chose the environment which gave the highest expected payoff in equilibrium. However, for the restricted rounds, denoted by $\mathrm{R}$ in the table, subjects' choices were surprisingly incorrect. Up to $80.00 \%$ of subjects chose the environment which did not provide the highest expected payoff in equilibrium. ${ }^{22}$ See Figure 4.

One possible concern is that experiment participants may have made their environment choices randomly. Since the variances of the environmental supports were large enough to prevent any player from having a winning probability close to $1,{ }^{23}$ and the monetary prize was not large enough to bring about a sizable loss in case of a wrong choice of environment, some of the subjects may simply have wanted to make random choices or to make "unusual" choices (unusual from their perspective). To address this concern, in Table 7 I provide summary statistics on the cohort of subjects who made the correct environment choices in all the unrestricted

\footnotetext{
${ }^{20}$ The undergraduate subjects chose the optimal environment in $30.44 \%$ of the restricted rounds, while the graduate subject chose it in $27.78 \%$ of the rounds.

${ }^{21}$ Under a null hypothesis of the same proportion of the correct environment choices, the $t$-statistic of the proportion difference between males and females is 0.2214 .

${ }^{22} \mathrm{My}$ model does not explicitly consider risk aversion, but if that were taken into account, the accuracy of the subjects' choices would be even lower, because they could have attained a more stable income stream by choosing the third-tier environment and a smaller investment level.

${ }^{23}$ The experiment was designed in a way that even when the third-tier player invests all the 40 tokens and the other players invest 0 tokens, it is still possible that the third-tier player loses due to the high variance of the environmental supports.
} 


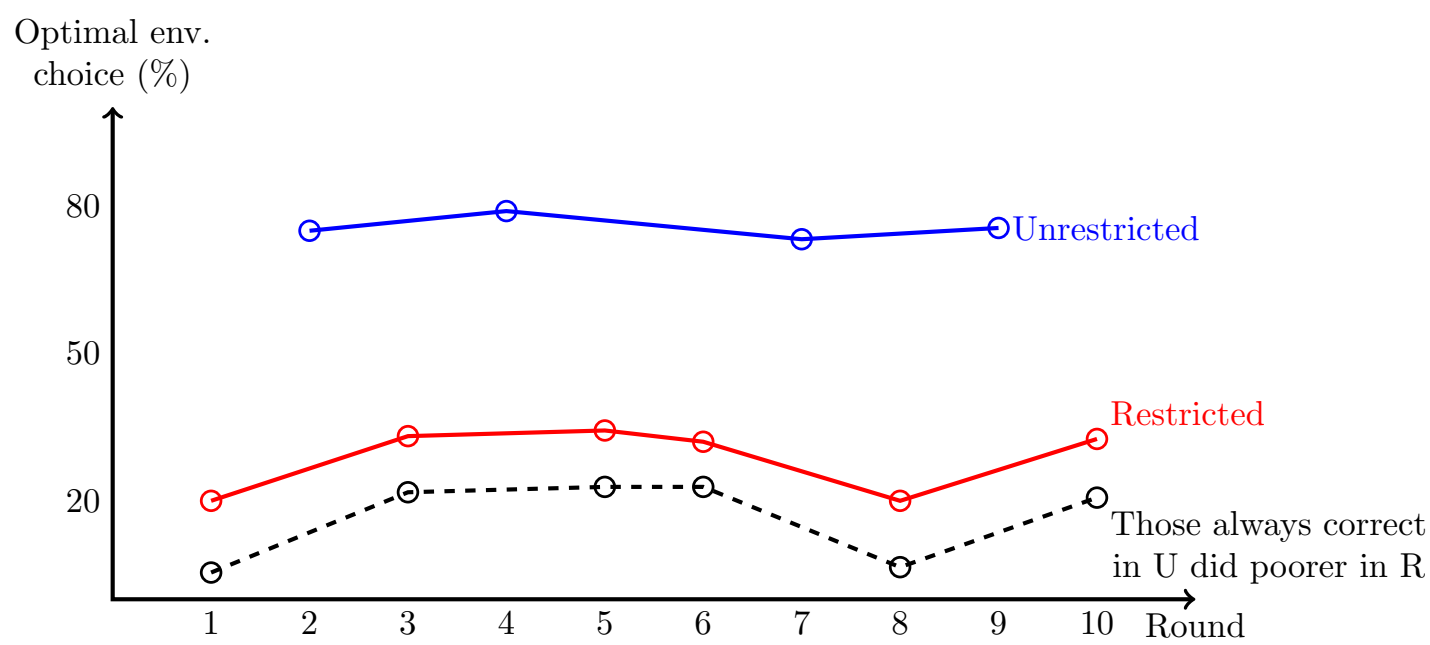

Figure 4:

The blue (red) line shows the proportion of subjects who chose the optimal environment in the unrestricted (restricted) rounds. In the unrestricted rounds, $73.14 \%$ or more of subjects chose the environment which gave the highest expected payoff in equilibrium. In the restricted rounds, however, subjects' choices were in general incorrect. Up to $80.00 \%$ of subjects chose the environment which did not provide the highest expected payoff in equilibrium. The dashed line shows the environment choices of the subjects who made the correct choice in all the unrestricted rounds; they did poorer in the restricted rounds.

rounds. I call them the ACU (Always Correct in the Unrestricted rounds) subjects. Note that they did poorer in the restricted rounds. See the dashed line in Figure 4. When it came to the investment choices, the subjects overinvested in every round on average. The last row in Tables 6 and 7 shows how subjects' investment choices differed from the Nash equilibrium investment given their environment choices. The ACU subjects' overinvestments were slightly smaller for all the rounds, but not significant enough to conclude that in general the ACU subjects exercised greater discretion in their individual investment choices.

\begin{tabular}{ccccccccccc}
\hline Round & 1 & 2 & 3 & 4 & 5 & 6 & 7 & 8 & 9 & 10 \\
\hline Game Type & $\mathrm{R}$ & $\mathrm{U}$ & $\mathrm{R}$ & $\mathrm{U}$ & $\mathrm{R}$ & $\mathrm{R}$ & $\mathrm{U}$ & $\mathrm{R}$ & $\mathrm{U}$ & $\mathrm{R}$ \\
SPNE & $(\mathrm{A}, 7)$ & $(\mathrm{C}, 16)$ & $(\mathrm{C}, 5)$ & $(\mathrm{B}, 17)$ & $(\mathrm{A}, 5)$ & $(\mathrm{C}, 3)$ & $(\mathrm{A}, 16)$ & $(\mathrm{B}, 7)$ & $(\mathrm{C}, 12)$ & $(\mathrm{A}, 3)$ \\
Mode (env. choice) & $\mathrm{C}$ & $\mathrm{C}$ & $\mathrm{B}$ & $\mathrm{B}$ & $\mathrm{B}$ & $\mathrm{A}$ & $\mathrm{A}$ & $\mathrm{C}$ & $\mathrm{C}$ & $\mathrm{B}$ \\
Correct decision (\%) & 5.43 & 100.00 & 21.74 & 100.00 & 22.83 & 22.83 & 100.00 & 6.52 & 100.00 & 20.65 \\
\hline Avg. inv. choice & 13.54 & 16.79 & 15.86 & 17.79 & 13.80 & 14.11 & 17.15 & 14.12 & 16.50 & 13.77 \\
Overinvestment & 3.89 & 4.89 & 8.88 & 4.58 & 6.89 & 10.09 & 5.25 & 4.58 & 7.34 & 9.32 \\
\hline
\end{tabular}

Table 7: Summary: The ACU Subjects Only

This table is the equivalent of Table 6 for the subjects who made the optimal choice of environment in all the unrestricted rounds. In each of the restricted rounds, the proportion of subjects who chose the optimal environment was rather small.

Some participants, especially the ACU subjects, may have set their own rule of thumb and strictly followed it. As stated in the previous section, any single statistic which summarizes the environmental distributions - either separately or jointly — cannot provide a consistent way 
of predicting the ranks of the expected payoffs in equilibrium. That is, no matter what a participant's simple rule of thumb was, ${ }^{24}$ their rule was wrong. Under this interpretation, the STT affected such participants even more severely because they consistently chose the second tier without contemplation and then backed up their (wrong) choices with overinvestment. The combination of these two mistakes, the wrong choices of environment and the overinvestments, caused them to have even lower expected payoffs.

Another observation may help to minimize any concern about whether the subjects used a simple, primitive rule of thumb to make their environment choices in earlier rounds and then modified their rule based on their previous experience. In 1,057 of the 1,400 "repeat" rounds (i.e., those other than the first unrestricted round and the first restricted round for each participant), the subject's choice of environment was consistent with that in their previous round. ${ }^{25}$ Among the 343 repeat rounds where they made a choice of environment that was inconsistent with their previous choice, 172 were from the first half of the eight repeat rounds, and 171 were from the second half. That is, I found no compelling evidence that subjects had modified their own decision rules about the choice of environment as a result of their experience on earlier rounds.

Though analysis of investment patterns was not the primary purpose of my experiments, at least two interesting observations are worth noting. One of these is that subjects' overinvestment levels were statistically significant at the $5 \%$ level. That is, subjects tended to overinvest when they believed either that they were likely to be a runner-up or that they had chosen an advantageous environment. A common finding in the rank-order tournament literature is that theory does a good job of predicting the average effort level in tournament experiments (Bull, Schotter, and Weigelt, 1987; Schotter and Weigelt, 1992; Harbring and Irlenbusch, 2011; Agranov and Tergiman, 2013). To the best of my knowledge, there have been no reports of overinvestment behavior with heterogeneous environmental supports in rank-order tournaments. ${ }^{26}$ The other interesting observation is that risk-seeking behavior after a previous loss was associated with a loss in competition, not in payoff. Note that in this experiment, it is possible for the player to lose the competition but have a positive payoff. Observations in other contexts of choice

\footnotetext{
${ }^{24}$ If a rule of thumb was used by an ACU, we cannot identify whether it was the mean or a lower bound of the environmental support distribution. Note that I set the environmental distributions in such a way that a distribution with a higher mean has a smaller variance, so that the STT could arise. In the experiment, all the environmental support distributions were uniform, so the ranks of the means are consistent with the ranks of the lower bounds of the uniform distributions.

25 (Un)restricted rounds were compared with previous (un)restricted ones.

${ }^{26} \mathrm{I}$ also looked at individuals who chose the second-best environment in at least one of the six restricted rounds and the second-tier environment in at least one of them, and found that the average investment level when they chose the second-tier environment (14.72 tokens) was higher than that when they chose the second-best environment (13.98 tokens). However, the difference is not statistically significant ( $t$-statistic: $0.8065, n_{1}=341, n_{2}=277$ ).
} 
under uncertainty (Weber and Zuchel, 2005; Langer and Weber, 2008; Andrade and Iyer, 2009) suggest that subjects become more risk averse after a "paper" loss in payoff. ${ }^{27}$ See Table 8. When the subjects in my experiments lost the competition in the previous round, they tended to overinvest by 1.93 tokens more than their previous overinvestment level, while they overinvested by 0.89 tokens less than their previous overinvestment level if they won the competition in the previous round. Subjects who lost in competition but gained in payoff in the previous round overinvested by 3.75 tokens more than their previous overinvestment level. On average, subjects who had a loss in payoff in the previous round overinvested by 2.70 tokens less than their previous overinvestment level, while those who gained in the previous round overinvested by 1.15 tokens more than their previous overinvestment level. However, this observation cannot be considered as a direct contribution to the literature on changes in risk attitude, because the structure of each round varied and each round could therefore be understood as a separate task.

\begin{tabular}{lc}
\hline & Changes in overinvestment ${ }^{*}$ at round $t$ \\
\hline Loss in competition in round $t-1$ & 1.93 \\
\hline Win in competition in round $t-1$ & -0.89 \\
\hline Loss in payoff in round $t-1$ & -2.70 \\
\hline Gain in payoff in round $t-1$ & 1.15 \\
\hline Loss in competition + gain in payoff in round $t-1$ & 3.75 \\
\hline Win in competition + loss in payoff in round $t-1$ & - \\
\hline
\end{tabular}

Table 8: Risk Attitude after a Previous Loss in Competition/Payoff

(*) The overinvestment level is defined as the actual investment level minus the optimal investment level given the subject's choice of environment. Therefore, changes in overinvestment may not directly imply changes in investment. Positive (negative) changes in overinvestment can be understood as the subjects' becoming risk-seeking (risk-averse) after a previous event.

The results of the follow-up survey are summarized in Table 9. The ACU subjects were more confident than the other subjects in their environment choices and more likely to report that the environment choices were easy. $76.09 \%$ of the ACU subjects answered that the closest description of their environment choices was "consistently right" or "in general right, except perhaps on only a few rounds," while only $58.33 \%$ of the non-ACU subjects chose one of those answers. Similarly, $54.35 \%$ of the ACU subjects found the environment choices "very easy" or "easy," while $34.52 \%$ of the others chose one of those answers. For similarly paired questions about

\footnotetext{
${ }^{27}$ Imas (2014) reports mixed findings in regard to the influence of prior losses on risk attitudes, and shows that individuals become risk averse after losses are realized while they become risk seeking if a loss has not been realized (that is, a paper loss). In my experiments, subjects were informed of their wins/losses in competition and their gains/losses in payoff after every round, but the payoffs were realized only at the end of the game. In the winner-takeall contest, the level of overinvestment may have captured subjects' attitude toward risk, since the optimal level of investment was calculated under an assumption of risk neutrality. If a subject invested more (less) than the optimal level, s/he decreased the expected payoff but expanded (reduced) the variance of possible earnings. Therefore, the positive (negative) changes in overinvestment imply that s/he became more risk-seeking (risk-averse).
} 
individual investment choices, there were no noticeable differences between the ACU subjects and non-ACU subjects, except that a larger proportion of the non-ACU subjects answered that the investment choices were easy, at the $10 \%$ level of statistical significance. I acknowledge that this between-group comparison may not have captured the individual heterogeneity in subjects' self-evaluation, so the simplest difference-in-differences estimator is also provided. Since the survey consisted of two sets of two questions each, I first calculated the difference between answers to the questions within each set. For example, if a subject answered that her environment choices were "consistently right" but her investment choices were "in general right, maybe one or two tokens more or less," then I counted her as having more confidence in her environment choices than in her investment choices. Similarly, if a subject answered that her environment choices were "easy" and her investment choices were "neither easy nor hard," then I counted her as considering that making the environment choices was the easier of the two tasks. Differences in these differences between the ACU subjects and the others are positive. That is, even after controlling for individual heterogeneity, we still observe that the ACU subjects were more likely than the others to feel that the environment choices were easier than the investment choices. These differences were tested under the null hypothesis of the same population mean.

The ACU subjects' stronger confidence in their environment choices could have lead to a larger loss in payoff. If a subject lost in competition, then there are three possible reasons why she lost: Her environment choice might be was wrong, she should have invested more, or she just had bad luck. Since the ACU subjects were more confident in environment choices, and unless they believe they are always unlucky, they would have been likely to consider investing more, or at least would have been tempted to blame themselves for not exerting more effort (i.e., for not investing enough) rather than contemplating their environment choices. The experimental evidence confirms this conjecture: On average, the ACU subjects' overinvestment level for the lost restricted rounds was 6.82 tokens, while that of the non-ACU subjects was 3.99 tokens. The difference was statistically significant ( $t$-test statistic: $3.6148, n_{1}=313, n_{2}=352$ ).

\section{Conclusions}

This paper's theoretical results can be summarized as follows: In a pairwise competition, only the first moment of the environmental supports matters, so the equilibrium payoffs reflect only the expected environmental supports. That is, a decision maker needs no information other than the expected environmental supports when there are only two life choices. With 


\begin{tabular}{|c|c|c|c|c|}
\hline \multirow{2}{*}{\multicolumn{2}{|c|}{ Survey Question }} & \multicolumn{2}{|c|}{ Positive answers $(\%)^{1}$} & \multirow{2}{*}{$\begin{array}{l}\text { differences } \\
(\% \text { points })\end{array}$} \\
\hline & & $\mathrm{ACU}$ & non-ACU & \\
\hline \multirow{2}{*}{\multicolumn{2}{|c|}{$\begin{array}{l}\text { 1.1. How confident in your environment choices? } \\
\text { 1.2. How confident in your investment choices? }\end{array}$}} & 76.09 & 58.33 & $17.75^{* * *}$ \\
\hline & & 65.22 & 63.10 & 2.12 \\
\hline \multirow{2}{*}{\multicolumn{2}{|c|}{$\begin{array}{l}\text { 2.1. How easy were environment choices? } \\
\text { 2.2. How easy were investment choices? }\end{array}$}} & 54.35 & 34.52 & $19.82^{* * *}$ \\
\hline & & 11.96 & 21.43 & $-9.47^{*}$ \\
\hline & \multicolumn{2}{|c|}{ Positive differences $(\%)^{2}$} & \multirow{2}{*}{\multicolumn{2}{|c|}{$\begin{array}{l}\text { difference-in-differences } \\
\text { (\% points })\end{array}$}} \\
\hline & $\mathrm{ACU}$ & non-ACU & & \\
\hline More confident in environment choices $^{3}$ & 31.52 & \multicolumn{2}{|c|}{26.19} & \multirow{2}{*}{$\begin{array}{l}5.33 \\
4.48^{* * *}\end{array}$} \\
\hline Easier in environment choices ${ }^{3}$ & 65.22 & 40.74 & & \\
\hline
\end{tabular}

Table 9: Summary: Survey

$*, * *, * * *: 10 \%, 5 \%, 1 \%$ level of statistical significance.

1. For questions 1.1 and 1.2, this shows the proportion of subjects who answered that the closest description of their environment choices (investment choices) was "consistently right" or "in general right, except perhaps on only a few rounds." For questions 2.1 and 2.2, it shows the proportion of subjects who answered "very easy" or "easy."

2. Questions 1.1 and 1.2, and questions 2.1 and 2.2, are paired, to capture the difference between answers in each pair.

3. After controlling for individual heterogeneity, we observe that the ACU subjects were more confident in their environment choices and found the environment choices easier. The differences were tested under the null hypothesis of the same population mean.

three players, the second moment of the environmental supports is also significant in determining the ranks of the equilibrium payoffs. The second-tier trap arises when the second-tier player is less competitive than the third-tier player in terms of the second moment and less competitive than the first-tier player in terms of the first moment. A sufficient condition for the STT is that the ex-ante advantage of the third-tier environment be at least as large as that of the second-tier environment. That is, a decision maker needs no information other than the expected environmental supports and the ex-ante advantages, and should choose the third-tier environment over the second-tier one when the ex-ante advantages of the second- and third-tier environments are similar. When it comes to the counterfactual analysis of an individual's life choices, it suggests that when the first-best option is unavailable, or the decision maker is not accepted for belonging to the first-best environment, it is wise to consider more than just the tiers. The experimental evidence strongly supports that players can easily be tempted to make the wrong decision in choosing their environments: The majority of subjects chose the environment with the largest available expectation of the environmental support, regardless of whether a simulator for payoff calculation was provided. A significant proportion of participants in my experiments failed to choose the better of two environments in terms of the maximum expected payoff in equilibrium when they were not given the option of choosing the best environment of the three, though they were fairly good at choosing the best environment. The results imply that following a simple rule of thumb based on one summary statistic, the mean, could result 
in the second-best player being worse off.

There are many potential directions for further research to extend this study. From the theoretical perspectives, I assumed in this study that players are identical in terms of the cost of their effort, so the first-stage matching becomes trivial. Considering a sequential competition with history-dependent human capital accumulation which affects the cost of the effort could be challenging but may address more of the issues that are relevant to real-life situations. Another key assumption of this paper was the winner-take-all payoff structure. It is worth investigating other forms of reward structures, for example, a combination of rewards and punishments as considered in Moldovanu, Sela, and Shi (2012) or Kamijo (2016), to see if those would yield the similar results. For further experiments, asking subjects to report the complete order of their environment preferences could help us relate those preferences to their effort choices. It would be worth doing a between-subject study with two treatments, one with the unrestricted framework and the other with the restricted framework, so that we could more rigorously identify the changes in investment behavior caused by the outcomes of previous events.

\section{Appendix A}

\section{Proof of existence of a Nash equilibrium:}

Note that players would not exert more effort than $\bar{e}$ such that $c(\bar{e})=w$, as that would definitely yield a negative expected payoff. Define $\mathbf{B R}(\mathbf{e})=\left(B R_{1}\left(e_{-1}\right), \ldots, B R_{n}\left(e_{-n}\right)\right)$, where $\mathbf{e}=\left(e_{1}, \ldots, e_{n}\right) \in[0, \bar{e}]^{n} \equiv S$. Since $S$ is a nonempty, closed, bounded, and convex subset of $\mathbb{R}^{n}$, and $\mathbf{B R}(\mathbf{e})$ is a upper-semi continuous self-map on $S$, by Kakutani's fixed point theorem there exists $\mathbf{e}^{*} \in S$ such that $\mathbf{B R}\left(\mathbf{e}^{*}\right)=\mathbf{e}^{*}$.

In the proof of Proposition 1, I will use the following observations and Lemma 1.

Observations. Suppose $g_{1}(\theta)$ and $g_{2}(\theta)$ are unimodal symmetric probability distributions with means $E\left(\theta_{1}\right)$ and $E\left(\theta_{2}\right)$, respectively. For Observations 1-4, suppose $E\left(\theta_{1}\right)>E\left(\theta_{2}\right)$.

1. Since $\int g_{1}(\theta) d \theta=\int g_{2}(\theta) d \theta=1, \int g_{1}(\theta) g_{2}(\theta) d \theta$ can be interpreted as a weighted average of $g_{1}$ weighted by $g_{2}$, or a weighted average of $g_{2}$ weighted by $g_{1}$.

2. For $G_{i}(x)=\int_{-\infty}^{x} g_{i}(\theta) d \theta, i=1,2, \int G_{i}(\theta) g_{i}(\theta) d \theta=\frac{1}{2}$.

3. $\int\left(G_{2}(\theta)-G_{1}(\theta)\right) g_{1}(\theta) d \theta>0$.

4. $\int\left(g_{1}(\theta)-g_{2}(\theta)\right) G_{1}(\theta) d \theta>0$. 
Suppose now that $E\left(\theta_{1}\right)=E\left(\theta_{2}\right)$.

5. Consider a location shift parameter $e \in \mathbb{R} . \int g_{1}(\theta-e) g_{2}(\theta) d \theta$ is maximized at $e=0$.

Proof: The first observation is trivial. The second observation follows from integration by parts: $\int G_{i}(\theta) g_{i}(\theta) d \theta=\left[G_{i}(\theta) G_{i}(\theta)\right]_{-\infty}^{\infty}-\int g_{i}(\theta) G_{i}(\theta) d \theta \Rightarrow 2 \int G_{i}(\theta) g_{i}(\theta) d \theta=1$. The third observation may be nontrivial. Since we assumed that the support distributions are symmetric and unimodal, there are only two possible cases with $E\left(\theta_{1}\right)>E\left(\theta_{2}\right)$ : either $\theta_{1}$ is first-order stochastically dominant (FOSD) over $\theta_{2}$, or $G_{1}$ and $G_{2}$ satisfy the single-crossing property, that is, there is $\theta^{*}$ such that for $\theta \geq \theta^{*}, G_{1}(\theta) \geq G_{2}(\theta)$, and for $\theta<\theta^{*}, G_{2}(\theta)>G_{1}(\theta)$. If $\theta_{1}$ is FOSD over $\theta_{2}$, then $G_{2}(\theta)-G_{1}(\theta) \geq 0$ for all $\theta$ and $G_{2}(\theta)-G_{1}(\theta)>0$ for some $\theta$, so $\int\left(G_{2}(\theta)-\right.$ $\left.G_{1}(\theta)\right) g_{1}(\theta) d \theta>0$. If $G_{2}(\theta)$ single-crosses $G_{1}(\theta)$ at $\theta^{*}$, then $\theta^{*}$ must be greater than $E\left(\theta_{1}\right)$. This is because $G_{i}\left(E\left(\theta_{i}\right)\right)=\frac{1}{2}, i=1,2$, and $G_{2}\left(E\left(\theta_{1}\right)\right)>G_{1}\left(E\left(\theta_{1}\right)\right)$. Then $\int_{-\infty}^{\theta^{*}} g_{1}(\theta) d \theta>$ $\int_{\theta^{*}}^{\infty} g_{1}(\theta) d \theta$, so $G_{2}(\theta)>G_{1}(\theta)$ for all $\theta<\theta^{*}$, and $\int_{-\infty}^{\theta^{*}}\left(G_{2}(\theta)-G_{1}(\theta)\right) d \theta>\int_{\theta^{*}}^{\infty}\left(G_{1}(\theta)-G_{2}(\theta)\right) d \theta$. Therefore, $\int\left(G_{2}(\theta)-G_{1}(\theta)\right) g_{1}(\theta) d \theta=\int_{-\infty}^{\theta^{*}}\left(G_{2}(\theta)-G_{1}(\theta)\right) g_{1}(\theta) d \theta-\int_{\theta^{*}}^{\infty}\left(G_{1}(\theta)-G_{2}(\theta)\right) g_{1}(\theta) d \theta>$ 0 . The fourth observation follows from the second and third observations. Using integration by parts, $\int\left(G_{2}(\theta)-G_{1}(\theta)\right) g_{1}(\theta) d \theta=\left[\left(G_{2}(\theta)-G_{1}(\theta)\right) G_{1}(\theta)\right]_{-\infty}^{\infty}-\int\left(g_{2}(\theta)-g_{1}(\theta)\right) G_{1}(\theta) d \theta=0+$ $\int\left(g_{1}(\theta)-g_{2}(\theta)\right) G_{1}(\theta) d \theta>0$. The fifth observation states that the integral of the product of two symmetric probability distributions is maximized when they peak at the same point. The firstorder condition on $\max _{e} \int g_{1}(\theta-e) g_{2}(\theta) d \theta$ is $\int-\frac{d g_{1}(\theta-e)}{d e} g_{2}(\theta) d \theta=\int \frac{d g_{1}(\tilde{\theta})}{d \tilde{\theta}} g_{2}(\tilde{\theta}+e) d \tilde{\theta}=0$. The first equality follows from the change of variables $\tilde{\theta}=\theta-e$. Since $\frac{d g_{1}(\theta)}{d \theta}$ is an odd function about $E(\theta)$, and $g_{2}(\theta)$ is an even function about $E(\theta), \int \frac{d g_{1}(\theta)}{d \theta} g_{2}(\theta) d \theta=0$. This observation is useful for evaluating $\int g_{1}(\theta-e) g_{2}(\theta) d \theta$ even when $E\left(\theta_{1}\right)$ and $E\left(\theta_{2}\right)$ are different. If $E\left(\theta_{1}\right)>E\left(\theta_{2}\right)$, then $\int g_{1}(\theta-e) g_{2}(\theta) d \theta$ is maximized when $e=E\left(\theta_{2}\right)-E\left(\theta_{1}\right)$. Also, $\int g_{1}(\theta-e) g_{2}(\theta) d \theta$ is smaller (greater) than $\int g_{1}(\theta) g_{2}(\theta) d \theta$ for $e>0$ (for $E\left(\theta_{2}\right)-E\left(\theta_{1}\right)<e<0$ ).

Lemma 1. If $E\left(\theta_{1}\right)>E\left(\theta_{2}\right)$, then $\int G_{2}(\theta) g_{1}(\theta) d \theta>\int G_{1}(\theta) g_{2}(\theta) d \theta$.

Proof: $\int G_{2}(\theta) g_{1}(\theta) d \theta>\int G_{1}(\theta) g_{1}(\theta) d \theta=\int G_{1}(\theta)\left(g_{1}(\theta)-g_{2}(\theta)+g_{2}(\theta)\right) d \theta=\int G_{1}(\theta) g_{2}(\theta) d \theta+$ $\int G_{1}(\theta)\left(g_{1}(\theta)-g_{2}(\theta)\right) d \theta>\int G_{1}(\theta) g_{2}(\theta) d \theta$, where the first inequality is from Observation 3 and the last inequality is from Observation 4.

\section{Proof of Proposition 1}

$[\Rightarrow]$ First, assume $E\left(\theta_{1}\right)>E\left(\theta_{2}\right)$. Since $E\left(\theta_{1}\right)>E\left(\theta_{2}\right)$ implies $\int G_{2}(x) g_{1}(x) d x>\int G_{1}(x) g_{2}(x) d x$ by Lemma 1, player 1's expected utility is always greater than or equal to that of player 2 at 
an equilibrium. Note that if the two players choose the same level of effort, then their expected rewards will be exactly the same as their ex-ante advantages, the probability of winning when everyone chooses no effort, that is, $E\left[R_{i}\left(e \mid \theta_{i}, \theta_{j}, e\right)\right]=\int G_{j}(\theta+e-e) g_{i}(\theta) d \theta=$ $\int G_{j}(\theta) g_{i}(\theta) d \theta$, where the value of the prize, $w$, is normalized to 1 . Then $E U_{1}^{*}\left(e_{1}^{*} \mid \theta_{1}, \theta_{2}, e_{2}^{*}\right)=$ $\max _{e_{1}} E U_{1}\left(e_{1} \mid \theta_{1}, \theta_{2}, e_{2}^{*}\right) \geq E U_{1}\left(e_{2}^{*} \mid \theta_{1}, \theta_{2}, e_{2}^{*}\right)=\int G_{2}(\theta) g_{1}(\theta) d \theta-c\left(e_{2}^{*}\right)>\int G_{1}(\theta) g_{2}(\theta) d \theta-$ $c\left(e_{2}^{*}\right)=E U_{2}^{*}\left(e_{2}^{*} \mid \theta_{2}, \theta_{1}, e_{2}^{*}\right)$. In other words, if player 2 chooses an effort level $e_{2}$, then player 1's expected utility can always be greater by exerting the same level of effort, resulting in a higher winning probability with the same cost of effort. Since this effort choice $e_{2}$ is attainable for player 1 , his maximized expected utility must be at least as large as the expected utility when he chooses $e_{2}$.

$[\Leftarrow]$ If $E\left[R_{1}\left(e_{1}^{*} \mid \theta_{1}, \theta_{2}, e_{2}^{*}\right)\right]-c\left(e_{1}^{*}\right)>E\left[R_{2}\left(e_{2}^{*} \mid \theta_{2}, \theta_{1}, e_{1}^{*}\right)\right]-c\left(e_{2}^{*}\right)$, then it is trivial to show that $E\left(\theta_{1}\right)>E\left(\theta_{2}\right)$ if we can prove that $e_{1}^{*} \geq e_{2}^{*}$ in equilibrium. Suppose for the sake of contradiction that $E\left(\theta_{1}\right) \leq E\left(\theta_{2}\right)$. Then $\left(e_{1}^{*}, e_{2}^{*}\right)$ is not an equilibrium, because this case $\left(E\left(\theta_{1}\right) \leq E\left(\theta_{2}\right)\right.$ and $\left.E\left[R_{1}\left(e_{1}^{*} \mid \theta_{1}, \theta_{2}, e_{2}^{*}\right)\right]-c\left(e_{1}^{*}\right)>E\left[R_{2}\left(e_{2}^{*} \mid \theta_{2}, \theta_{1}, e_{1}^{*}\right)\right]-c\left(e_{2}^{*}\right)\right)$ holds only when $e_{2}^{*}>e_{1}^{*}$. Thus all that remains is to show that $e_{1}^{*} \geq e_{2}^{*}$ in equilibrium.

For the two-player competition, player $i$ 's first-order condition is $\int g_{j}\left(\theta+e_{i}-e_{j}^{*}\right) g_{i}(\theta) d \theta=$ $c^{\prime}\left(e_{i}\right)$, where the value of the prize, $w$, is normalized to 1 . Similarly, player $j$ 's first-order condition is $\int g_{i}\left(\theta+e_{j}-e_{i}^{*}\right) g_{j}(\theta) d \theta=c^{\prime}\left(e_{j}\right)$. When $e_{i}=e^{*}=e_{j}$, both first-order conditions are satisfied. Therefore $\left(e_{i}^{*}, e_{j}^{*}\right)$ with $e_{i}^{*}=e_{j}^{*}$ is an equilibrium. This is not necessarily the only Nash equilibrium, because we have not checked whether $\left(e_{1}^{*}, e_{2}^{*}\right)$ with $e_{1}^{*} \neq e_{2}^{*}$ can be an equilibrium.

My goal is to show that $e_{2}^{*}>e_{1}^{*}$ cannot be an equilibrium. Suppose for the sake of contradiction that $\left(\hat{e}_{1}, \hat{e}_{2}\right)$ with $\hat{e}_{2}>\hat{e}_{1}$ is an equilibrium. Let $\varepsilon=\hat{e}_{2}-\hat{e}_{1}>0$. Since $c^{\prime}(\cdot)$ is monotone increasing, $\int g_{1}(\theta+\varepsilon) g_{2}(\theta) d \theta=c^{\prime}\left(\hat{e}_{2}\right)>c^{\prime}\left(\hat{e}_{1}\right)=\int g_{2}(\theta-\varepsilon) g_{1}(\theta) d \theta$. $\hat{e}_{2}>\hat{e}_{1}$ also implies that when player 2 chooses $\hat{e}_{1}$, the marginal benefit is greater than the marginal cost, that is, $\int g_{1}(\theta+$ $\left.\hat{e}_{1}-\hat{e}_{1}\right) g_{2}(\theta) d \theta \geq c^{\prime}\left(\hat{e}_{1}\right)$, or $\int g_{1}(\theta) g_{2}(\theta) d \theta \geq c^{\prime}\left(\hat{e}_{1}\right)=\int g_{2}(\theta-\varepsilon) g_{1}(\theta) d \theta$. This is a contradiction for $\varepsilon \in\left(0,2\left(E\left(\theta_{1}\right)-E\left(\theta_{2}\right)\right)\right)$. Note that $\int g_{2}(\theta-\varepsilon) g_{1}(\theta) d \theta$ is maximized at $\varepsilon=E\left(\theta_{1}\right)-E\left(\theta_{2}\right)$, by the proof of Observation 5 . When $\varepsilon=2\left(E\left(\theta_{1}\right)-E\left(\theta_{2}\right)\right)$, by symmetry $\int g_{2}(\theta-\varepsilon) g_{1}(\theta) d \theta=$ $\int g_{2}(\theta) g_{1}(\theta) d \theta$. Thus for $\varepsilon \in\left(0,2\left(E\left(\theta_{1}\right)-E\left(\theta_{2}\right)\right)\right), \int g_{2}(\theta-\varepsilon) g_{1}(\theta) d \theta>\int g_{2}(\theta) g_{1}(\theta) d \theta$. Similarly, when player 1 chooses $\hat{e}_{2}$, the marginal benefit is smaller than the marginal cost, that is, $\int g_{2}\left(\theta+\hat{e}_{2}-\hat{e}_{2}\right) g_{1}(\theta) d \theta \leq c^{\prime}\left(\hat{e}_{2}\right)$, or $\int g_{2}(\theta) g_{1}(\theta) d \theta \leq c^{\prime}\left(\hat{e}_{2}\right)=\int g_{1}(\theta+\varepsilon) g_{2}(\theta) d \theta$. This is a contradiction for $\varepsilon>2\left(E\left(\theta_{1}\right)-E\left(\theta_{2}\right)\right)$, because $\int g_{2}(\theta) g_{1}(\theta) d \theta>\int g_{1}(\theta+\varepsilon) g_{2}(\theta) d \theta$. It is also a contradiction for $\varepsilon=2 E\left(\theta_{1}\right)-2 E\left(\theta_{2}\right)$, because $c^{\prime}\left(\hat{e}_{2}\right)=\int g_{1}\left(\theta+2 E\left(\theta_{1}\right)-2 E\left(\theta_{2}\right)\right) g_{2}(\theta) d \theta=$ 
$\int g_{2}\left(\theta-2 E\left(\theta_{1}\right)+2 E\left(\theta_{2}\right)\right) g_{1}(\theta) d \theta=c^{\prime}\left(\hat{e}_{1}\right)$ but $c^{\prime}\left(\hat{e}_{2}\right)>c^{\prime}\left(\hat{e}_{1}\right)$. The second equality follows from the change of variables $\theta^{\prime}=\theta+2 E\left(\theta_{1}\right)-2 E\left(\theta_{2}\right)$. Therefore, in equilibrium $e_{1}^{*} \geq e_{2}^{*}$.

Lemma 2. Suppose $E\left(\theta_{1}\right)=E\left(\theta_{2}\right)=E\left(\theta_{3}\right)=\mu$, and $\theta_{2}^{\prime}$ is a mean-preserving spread of $\theta_{2}$. Then $\int G_{1}(\theta)^{2}\left(g_{2}(\theta)-g_{2}^{\prime}(\theta)\right) d \theta<0$ and $\int G_{1}(\theta) G_{3}(\theta)\left(g_{2}(\theta)-g_{2}^{\prime}(\theta)\right) d \theta<0$.

Proof: First, I show that $\int G_{1}(\theta) g_{2}^{\prime}(\theta) d \theta=\int G_{1}(\theta) g_{2}(\theta) d \theta=1 / 2$, to illustrate the tools I used in the proof of this lemma. $G_{1}(\theta)$ is mirror-imaged about $(\mu, 1 / 2)$, that is, for any $\epsilon>0, G_{1}(\mu-\epsilon)=1-G_{1}(\mu+\epsilon)$. In addition, $g_{2}(\theta)$ and $g_{2}^{\prime}(\theta)$ are symmetric about $\mu$, that is, for any $\epsilon>0, g_{2}(\mu-\epsilon)=g_{2}(\mu+\epsilon)$ and $g_{2}^{\prime}(\mu-\epsilon)=g_{2}^{\prime}(\mu+\epsilon)$. Then $\int G_{1}(\theta) g_{2}(\theta) d \theta=$ $\int_{-\infty}^{\mu} G_{1}(\theta) g_{2}(\theta) d \theta+\int_{\mu}^{\infty} G_{1}(\theta) g_{2}(\theta) d \theta=\int_{0}^{\infty} G_{1}(\mu-\epsilon) g_{2}(\mu-\epsilon) d \epsilon+\int_{0}^{\infty} G_{1}(\mu+\epsilon) g_{2}(\mu+\epsilon) d \epsilon=$ $\int_{0}^{\infty} G_{1}(\mu-\epsilon) g_{2}(\mu+\epsilon) d \epsilon+\int_{0}^{\infty} G_{1}(\mu+\epsilon) g_{2}(\mu+\epsilon) d \epsilon=\int_{0}^{\infty}\left(G_{1}(\mu-\epsilon)+G_{1}(\mu+\epsilon)\right) g_{2}(\mu+\epsilon) d \epsilon=$ $\int_{0}^{\infty} g_{2}(\mu+\epsilon) d \epsilon=\frac{1}{2}$. This holds for any symmetric probability distribution about $\mu$, including $g_{2}^{\prime}(\theta)$. Thus, $\int G_{1}(\theta) g_{2}^{\prime}(\theta) d \theta=\frac{1}{2}$.

$G_{1}(\theta)^{2}$ is not mirror-imaged about $(\mu, 1 / 2)$. For any $\epsilon>0, G_{1}(\mu-\epsilon)+G_{1}(\mu+\epsilon)=1$, but $G_{1}(\mu-\epsilon)^{2}+G_{1}(\mu+\epsilon)^{2}<1$. (For example, consider a uniform distribution from 0 to 1 whose mean is 0.5 . Then $(0.5-0.2)^{2}=0.09<1-(0.5+0.2)^{2}=0.51$.) When $\theta_{2}^{\prime}$ is a mean-preserving spread of $\theta_{2}$, there must be $d>0$ such that $g_{2}(\theta) \geq g_{2}^{\prime}(\theta)$ for $\theta \in[\mu-d, \mu+d]$, and $g_{2}^{\prime}(\theta)>g_{2}(\theta)$ for $\theta>\mu+d$ and $\theta<\mu-d$. Define $h(\theta)=g_{2}(\theta)-g_{2}^{\prime}(\theta)$. Then $h(\theta)$ is symmetric about $\mu$ and $\lim _{\theta \rightarrow-\infty} h(\theta)=\lim _{\theta \rightarrow \infty} h(\theta)=h(\mu-d)=h(\mu+d)=0 . h(\theta)$ is positive for $\theta \in[\mu-d, \mu+d]$ and negative otherwise. Moreover, $\int_{\mu}^{\infty} h(\theta) d \theta=\int_{\mu}^{\infty} g_{2}(\theta) d \theta-\int_{\mu}^{\infty} g_{2}^{\prime}(\theta) d \theta=\frac{1}{2}-\frac{1}{2}=0$. Thus,

$$
\begin{aligned}
& \int G_{1}(\theta)^{2}\left(g_{2}(\theta)-g_{2}^{\prime}(\theta)\right) d \theta=\int G_{1}(\theta)^{2} h(\theta) d \theta=\int_{-\infty}^{\mu} G_{1}(\theta)^{2} h(\theta) d \theta+\int_{\mu}^{\infty} G_{1}(\theta)^{2} h(\theta) d \theta \\
& =\int_{0}^{\infty} G_{1}(\mu-\epsilon)^{2} h(\mu-\epsilon) d \epsilon+\int_{0}^{\infty} G_{1}(\mu+\epsilon)^{2} h(\mu+\epsilon) d \epsilon \\
& =\int_{0}^{\infty}\left(G_{1}(\mu-\epsilon)^{2}+G_{1}(\mu+\epsilon)^{2}\right) h(\mu+\epsilon) d \epsilon<\int_{0}^{\infty} h(\mu+\epsilon) d \epsilon=\int_{\mu}^{\infty} h(\theta) d \theta=0 .
\end{aligned}
$$

Here $G_{1}(\theta)^{2}=G_{1}(\theta) G_{1}(\theta)$ could be replaced with the product of two different cumulative distributions. Suppose $\theta_{3}$ is another symmetric probability distribution with mean $\mu$. Then $\int G_{1}(\theta) G_{3}(\theta)\left(g_{2}(\theta)-g_{2}^{\prime}(\theta)\right) d \theta<0$. The proof is analogous after replacing $G_{1}(\mu-\epsilon)^{2}$ with $G_{1}(\mu-\epsilon) G_{3}(\mu-\epsilon)$.

\section{Proof of Proposition 2}

Let $\theta_{i}^{\prime}$ be a mean-preserving spread of $\theta_{i}$. Denote the pdf of $\theta_{i}^{\prime}$ by $g_{i}^{\prime}(\theta)$, so $\int \theta g_{i}(\theta) d \theta=E\left(\theta_{i}\right)=$ 
$\int \theta g_{i}^{\prime}(\theta) d \theta$ and $\int \theta^{2} g_{i}(\theta) d \theta<\int \theta^{2} g_{i}^{\prime}(\theta) d \theta$. Also, let $A_{j}^{i}$ be the ex-ante advantage of player $j$ with $\theta_{i}^{\prime}$, that is, $A_{i}^{i}=\int G_{j}(\theta) G_{k}(\theta) g_{i}^{\prime}(\theta) d \theta$, and $A_{j}^{i}=\int G_{i}^{\prime}(\theta) G_{k}(\theta) g_{j}(\theta) d \theta$, where $i, j$, and $k$ are distinct.

First, I show that $\int G_{j}(\theta) G_{k}(\theta)\left(g_{i}(\theta)-g_{i}^{\prime}(\theta)\right) d \theta<0$, or $A_{i}<A_{i}^{i}$, where $i=2,3$. By Lemma 2, we know it is true when $E\left(\theta_{i}\right)=E\left(\theta_{j}\right)=E\left(\theta_{k}\right)=\mu$. The one further step needed to complete this proof is to show that $G_{j}\left(E\left(\theta_{i}\right)-\epsilon\right) G_{k}\left(E\left(\theta_{i}\right)-\epsilon\right)+G_{j}\left(E\left(\theta_{i}\right)+\epsilon\right) G_{k}\left(E\left(\theta_{i}\right)+\epsilon\right)<1$. This will consist of three cases, but the main argument will be similar to the following: "For strictly positive $A, B, C$ and $D$, if $A+C \leq 1$ and $B+D \leq 1$, then $A B+C D<1$." (The proof of this claim is as follows: $A B+C D \leq A(1-D)+(1-A) D$. Redefine $A=0.5+\delta, \delta \in(-0.5,0.5)$, and $D=0.5+\eta, \eta \in(-0.5,0.5)$. Then $A(1-D)+(1-A) D=(0.5+\delta)(0.5-\eta)+(0.5-\delta)(0.5+\eta)=$ $0.5-2 \delta \eta<1$.

Consider a mean-preserving spread of $\theta_{3}$, whose mean $E\left(\theta_{3}\right)$ is the smallest of the three. For $\epsilon \in\left(0, E\left(\theta_{1}\right)-E\left(\theta_{3}\right)\right], G_{2}\left(E\left(\theta_{3}\right)-\epsilon\right) G_{1}\left(E\left(\theta_{3}\right)-\epsilon\right)+G_{2}\left(E\left(\theta_{3}\right)+\epsilon\right) G_{1}\left(E\left(\theta_{3}\right)+\epsilon\right)<1$ holds, because even at $\epsilon=E\left(\theta_{1}\right)-E\left(\theta_{3}\right), G_{2}\left(E\left(\theta_{3}\right)+\epsilon\right)<1$ and $G_{1}\left(E\left(\theta_{3}\right)+\epsilon\right)=1 / 2$. Therefore, $G_{2}\left(E\left(\theta_{3}\right)+\epsilon\right) G_{1}\left(E\left(\theta_{3}\right)+\epsilon\right)<1 / 2$. For $\epsilon>E\left(\theta_{3}\right)-E\left(\theta_{1}\right)$, and since $\left(E\left(\theta_{3}\right)+\right.$ $\epsilon)-E\left(\theta_{1}\right)<E\left(\theta_{1}\right)-\left(E\left(\theta_{3}\right)-\epsilon\right)$, that is, $E\left(\theta_{3}\right)+\epsilon$ is closer to $E\left(\theta_{1}\right)$ than $E\left(\theta_{3}\right)-\epsilon$, we find $G_{1}\left(E\left(\theta_{3}\right)+\epsilon\right)-1 / 2<1 / 2-G_{1}\left(E\left(\theta_{3}\right)-\epsilon\right)$, or $G_{1}\left(E\left(\theta_{3}\right)-\epsilon\right)+G_{1}\left(E\left(\theta_{3}\right)+\epsilon\right)<1$. This also holds for $G_{2}$, so $G_{2}\left(E\left(\theta_{3}\right)-\epsilon\right)+G_{2}\left(E\left(\theta_{3}\right)+\epsilon\right)<1$. Therefore, $G_{2}\left(E\left(\theta_{3}\right)-\epsilon\right) G_{1}\left(E\left(\theta_{3}\right)-\epsilon\right)+$ $G_{2}\left(E\left(\theta_{3}\right)+\epsilon\right) G_{1}\left(E\left(\theta_{3}\right)+\epsilon\right)<1$.

Next, consider a mean-preserving spread of $\theta_{2}$, whose mean $E\left(\theta_{2}\right)$ is between $E\left(\theta_{3}\right)$ and $E\left(\theta_{1}\right)$. For $\epsilon \in\left(0, \min \left\{E\left(\theta_{1}\right)-E\left(\theta_{2}\right), E\left(\theta_{2}\right)-E\left(\theta_{3}\right)\right\}\right], G_{3}\left(E\left(\theta_{2}\right)-\epsilon\right) G_{1}\left(E\left(\theta_{2}\right)-\epsilon\right)+G_{3}\left(E\left(\theta_{2}\right)+\right.$ $\epsilon) G_{1}\left(E\left(\theta_{2}\right)+\epsilon\right)<1$, because even at $\epsilon=\min \left\{E\left(\theta_{1}\right)-E\left(\theta_{2}\right), E\left(\theta_{2}\right)-E\left(\theta_{3}\right)\right\}, G_{3}\left(E\left(\theta_{2}\right)+\epsilon\right)<1$ and $G_{1}\left(E\left(\theta_{2}\right)+\epsilon\right) \leq 1 / 2$ (with equality when $E\left(\theta_{1}\right)-E\left(\theta_{2}\right) \leq E\left(\theta_{2}\right)-E\left(\theta_{3}\right)$ ). Therefore, $G_{3}\left(E\left(\theta_{2}\right)+\epsilon\right) G_{1}\left(E\left(\theta_{2}\right)+\epsilon\right)<1 / 2$. For $\epsilon>\min \left\{E\left(\theta_{1}\right)-E\left(\theta_{2}\right), E\left(\theta_{2}\right)-E\left(\theta_{3}\right)\right\}$, similarly to the case above, $G_{1}\left(E\left(\theta_{2}\right)-\epsilon\right)+G_{1}\left(E\left(\theta_{2}\right)+\epsilon\right)<1$.

This approach will not work for $\theta_{1}$, whose mean $E\left(\theta_{1}\right)$ is the highest of the three. For any $\epsilon>0, G_{3}\left(E\left(\theta_{1}\right)-\epsilon\right)+G_{3}\left(E\left(\theta_{1}\right)+\epsilon\right)>1$ and $G_{2}\left(E\left(\theta_{1}\right)-\epsilon\right)+G_{2}\left(E\left(\theta_{1}\right)+\epsilon\right)>1$. In this case, $G_{3}\left(E\left(\theta_{1}\right)-\epsilon\right) G_{2}\left(E\left(\theta_{1}\right)-\epsilon\right)+G_{3}\left(E\left(\theta_{1}\right)+\epsilon\right) G_{2}\left(E\left(\theta_{1}\right)+\epsilon\right)$ can be larger or smaller than 1.

Next, I show that $A_{j}^{i}-A_{j}=\int\left(G_{i}^{\prime}(x)-G_{i}(x)\right) G_{k}(x) g_{j}(x) d x<0$. Since $\theta_{i}^{\prime}$ is a mean-preserving spread of $\theta_{i}, G_{i}^{\prime}(x)-G_{i}(x) \geq 0$ for all $x \leq E\left(\theta_{i}\right)$, and $G_{i}^{\prime}(x)-G_{i}(x) \leq 0$ for all $x>E\left(\theta_{i}\right)$. Define $H(\theta)=G_{i}^{\prime}(\theta)-G_{i}(\theta)$. Then $H(\theta)$ is symmetric about $E\left(\theta_{i}\right)$, so $H\left(E\left(\theta_{i}\right)-\epsilon\right)=-H\left(E\left(\theta_{i}\right)+\epsilon\right)$ 
for any $\epsilon>0$. Thus,

$$
\begin{aligned}
& \int\left(G_{3}^{\prime}(\theta)-G_{3}(\theta)\right) G_{1}(\theta) g_{2}(\theta) d \theta=\int H(\theta) G_{1}(\theta) g_{2}(\theta) d \theta \\
& =\int_{-\infty}^{E\left(\theta_{3}\right)} H(\theta) G_{1}(\theta) g_{2}(\theta) d \theta+\int_{E\left(\theta_{3}\right)}^{\infty} H(\theta) G_{1}(\theta) g_{2}(\theta) d \theta \\
& =\int_{0}^{\infty} H\left(E\left(\theta_{3}\right)-\epsilon\right) G_{1}\left(E\left(\theta_{3}\right)-\epsilon\right) g_{2}\left(E\left(\theta_{3}\right)-\epsilon\right) d \epsilon+\int_{0}^{\infty} H\left(E\left(\theta_{3}\right)+\epsilon\right) G_{1}\left(E\left(\theta_{3}\right)+\epsilon\right) g_{2}\left(E\left(\theta_{3}\right)+\epsilon\right) d \epsilon \\
& \leq \int_{0}^{\infty} \underbrace{\left(H\left(E\left(\theta_{3}\right)-\epsilon\right)+H\left(E\left(\theta_{3}\right)+\epsilon\right)\right)}_{=0} \max \left\{G_{1}\left(E\left(\theta_{3}\right)-\epsilon\right) g_{2}\left(E\left(\theta_{3}\right)-\epsilon\right), G_{1}\left(E\left(\theta_{3}\right)+\epsilon\right) g_{2}\left(E\left(\theta_{3}\right)+\epsilon\right)\right\} d \theta \\
& =0,
\end{aligned}
$$

where equality holds only when $G_{1}\left(E\left(\theta_{3}\right)-\epsilon\right) g_{2}\left(E\left(\theta_{3}\right)-\epsilon\right)=G_{1}\left(E\left(\theta_{3}\right)+\epsilon\right) g_{2}\left(E\left(\theta_{3}\right)+\epsilon\right)$ for every $\epsilon$, which is impossible with different means. Therefore, $\int\left(G_{3}^{\prime}(\theta)-G_{3}(\theta)\right) G_{1}(\theta) g_{2}(\theta) d \theta<0$, or $A_{1}^{3}-A_{1}<0 . A_{2}^{3}<A_{2}, A_{1}^{2}<A_{1}$ and $A_{3}^{2}<A_{3}$ can be shown analogously.

The marginal benefit for player $i$ which would accrue from increasing the level of effort is $\frac{\partial \int G_{j}\left(\theta+e_{i}-e_{j}\right) G_{k}\left(\theta+e_{i}-e_{k}\right) g_{i}(\theta) d \theta}{\partial e_{i}}$ (if $w$ is normalized to 1 ), and the marginal cost of increasing the level of effort is $c^{\prime}\left(e_{i}\right)$. While the marginal cost is independent of the spread of the support distribution, the marginal benefit decreases, as $\theta_{i}$ has a larger variance. To prove this, it suffices to show that $\int G_{j}\left(\theta+e_{i}-e_{j}\right) G_{k}\left(\theta+e_{i}-e_{k}\right)\left(g_{i}(\theta)-g_{i}^{\prime}(\theta)\right) d \theta$ is increasing in $e_{i}$. This is straightforward, because $G_{j}\left(\theta+e_{i}-e_{j}\right) G_{k}\left(\theta+e_{i}-e_{k}\right)$ is increasing in $e_{i}$, and $g_{i}(\theta)-g_{i}^{\prime}(\theta)$ does not vary with $e_{i}$. Therefore, $\frac{\partial \int G_{j}\left(\theta+e_{i}-e_{j}\right) G_{k}\left(\theta+e_{i}-e_{k}\right)\left(g_{i}(\theta)-g_{i}^{\prime}(\theta)\right) d \theta}{\partial e_{i}}=$ $\frac{\partial \int G_{j}\left(\theta+e_{i}-e_{j}\right) G_{k}\left(\theta+e_{i}-e_{k}\right) g_{i}(\theta) d \theta}{\partial e_{i}}-\frac{\partial \int G_{j}\left(\theta+e_{i}-e_{j}\right) G_{k}\left(\theta+e_{i}-e_{k}\right) g_{i}^{\prime}(\theta) d x}{\partial e_{i}}>0$. Since the marginal benefit of effort is smaller while the marginal cost of effort is the same, $e_{i}^{\prime *}$ has to decrease. This argument also holds for $e_{j}^{\prime *}, j \neq i$. Intuitively, a mean-preserving spread renders the effect of every player's effort less influential, so they would exert less effort.

Since a mean-preserving spread of $\theta_{i}$ drives increases in $A_{i}$, and since $e^{*}$ decreases, as does $A_{j}$ for $j \neq i$, it is natural for $E U_{i}^{\prime *}\left(e_{i}^{*} \mid \theta_{1}, \theta_{2}, \theta_{3}, e_{-i}^{*}\right)$ to increase. Even though the direction of $E U_{j}^{\prime *}$ is hard to determine from $e_{j}^{*}$ and $A_{j}^{i}$, we can at least conclude that $E U_{j}^{\prime *}$ cannot be strictly larger than $E U_{j}^{*}$. Suppose for the sake of contradiction that $E U_{j}^{\prime *}$ is strictly greater than $E U_{j}^{*}$. That would mean that player $j$ could attain a higher expected payoff with a lower ex-ante advantage. Since $e_{j}^{*} \leq e_{j}^{*}, e_{j}^{\prime *}$ is a feasible effort choice for player $j$. Therefore, $e_{j}^{*}$ cannot be an equilibrium effort for player $j$.

\section{Proof of Corollary 1}


Consider a shift of the location parameter, $\theta_{2} \stackrel{d}{=} \tilde{\theta}_{3}+x$, for a deterministic $x>0$, so that $E\left(\theta_{2}\right)-E\left(\tilde{\theta}_{3}\right)=x>0$. Then $E\left[R_{2}\left(e_{2}^{*} \mid \theta_{1}, \theta_{2}, \tilde{\theta}_{3}, e_{-2}^{*}\right)\right]-c\left(e_{2}^{*}\right)>E\left[R_{3}\left(e_{3}^{*} \mid \theta_{1}, \theta_{2}, \tilde{\theta}_{3}, e_{-3}^{*}\right)\right]-c\left(e_{3}^{*}\right)$, because the second-tier player has already been given a positive amount of "effort," $x$, for free. Player 2's expected payoff can be at least as large as that of player 3 by choosing $e_{3}^{*}$. By Proposition 2, $E U_{3}^{*}$ is increasing and $E U_{2}^{*}$ is decreasing in $\operatorname{Var}\left(\theta_{3}\right)$. As $\operatorname{Var}\left(\theta_{3}\right)$ goes to infinity, all the effort choices approach 0 , and $A_{3}$ approaches 0.5 , so $E U_{3}^{*}$ eventually exceeds $E U_{2}^{*}$. Any finite level of effort $e \in\left[0, c^{-1}(w)\right]$, where $c^{-1}(\cdot)$ is an inverse function of $c(\cdot)$, cannot affect the probability of winning as the variance of one of the environmental supports goes to infinity, holding other two variances fixed. As the variance of $\theta_{3}$ goes to infinity, each player's marginal benefit of effort approaches zero as shown in the proof of Proposition 2. As they exerts less effort, the expected payoff is more dependent to the ex-ante advantage. As $\operatorname{Var}\left(\theta_{3}\right)$ goes to infinity, the probability that the realization of $\theta_{3}$ is "extremer" than the realizations of $\theta_{1}$ and $\theta_{2}$, that is, with $50 \%$ of chance the realization of $\theta_{3}$ is the largest, and with another $50 \%$ of change it is the smallest.

\section{Proof of Proposition 3}

First, consider the case where the environmental support distributions of the second and third tiers are identical. Denote the equilibrium effort choices in this case by $e^{p}=\left(e_{1}^{p}, e_{2}^{p}, e_{3}^{p}\right)$. Then it is obvious that $A_{2}=A_{3}$ and $e_{1}^{p} \geq e_{2}^{p}=e_{3}^{p}$, just as in the pairwise competition. If the ex-ante advantages of the second and third players are also identical, they will have the same expected payoff, that is, $E U_{2}\left(e_{2}^{p} \mid \theta_{1}, \theta_{2}, \theta_{3}, e_{-2}^{p}\right)=E U_{3}\left(e_{3}^{p} \mid \theta_{1}, \theta_{2}, \theta_{3}, e_{-3}^{p}\right)$. Next, consider increasing the mean of $\theta_{2}$ and decreasing the variance of $\theta_{2}$ in such a way that $E\left(\theta_{2}\right)>E\left(\theta_{3}\right)$ while keeping $A_{2}=A_{3}$. The smaller variance of the support will increase the marginal benefit of increasing the level of effort, as shown in the proof of Proposition 2. Since the support distribution is symmetric and unimodal, a positive location shift will cause the marginal benefit of increasing the effort evaluated at $e_{2}^{p}$ to increase (or to remain the same when the distribution is uniform). Therefore, the second-tier player will choose the higher level of effort. By Proposition 2, $e_{3}^{p}$ will decrease but the expected reward will be unchanged. Hence the second-tier player will have a smaller equilibrium payoff, and a support distribution that has a larger mean and a smaller variance. 


\section{Appendix B}

\section{Sample Experimental Instructions}

[Have the participants sign in. Open the tutorial program.]

Welcome. This tutorial consists of instructions for the experiment, followed by a screening test and two practice rounds. Your close attention is important to this study. Please read the instructions very carefully.

\section{General description:}

During the experiment, you will earn "points." Your goal is to earn as many points as possible. The points will be converted to U.S. dollars at the end of the experiment, at the rate of 1,000 points $=1$ USD. As a reward for your participation, you will be given 7,000 points at the start of the experiment. The exact amount you will earn depends on your decisions, the decisions of two virtual competitors, and some luck. The experiment will consist of 10 rounds, each with two phases. In each round, you will have 40 virtual tokens. In general, your task is to choose an "environment" in the first phase of each round, and then to choose an amount of "individual investment" in the second phase. The details follow.

\section{In the first phase of each round:}

You will choose one of three environments, each of which is described by a range of numbers. At the end of the round, your chosen environment will randomly determine the amount of "environmental support" you receive. For example, if you choose an environment described as $[3.44,11.87]$, then at the end of the round your environment will randomly pick a number between 3.44 and 11.87, which will be the environmental support. No two players can choose the same environment, so your two competitors cannot choose the same environment you chose. (Therefore, after one computer competitor chooses an environment, the other competitor will be assigned the remaining one.) In some rounds, one environment may already have been chosen by one of your competitors. In that case, you will choose one of the remaining two environments.

\section{In the second phase of each round:}

You will choose an amount of individual investment with the 40 tokens. Your "output" is the sum of the environmental support you receive and your chosen individual investment. 


\section{Calculating your payoff:}

If your output is the largest of the three players, you will win 1600 points; otherwise, you will win nothing. If you invest $X$ tokens in the round, the cost of investment in terms of points is $X^{2}$, the square of $X$. The amount of your unused tokens, $40-X$, will be converted to points at the rate of 1 token $=10$ points. Thus, your payoff for the round will be $1600-X^{2}+(40-X) * 10$ points if you win, and $-X^{2}+(40-X) * 10$ points if you lose. Examples follow.

\section{Examples:}

Suppose you invest 10 tokens and your environmental support is 7.58. If your output, 17.58, is the largest of the three players, you will win and earn 1600 points, but you will pay 100 points (the square of 10) as the cost of investment. Your 30 unused tokens will be converted to 300 points. Therefore, your payoff for the round will be $1600-10^{2}+(40-10) * 10=$ $1600-100+300=1800$ points if you win. If you lose, it will be $-100+300=200$ points.

Suppose instead that you invest 30 tokens. You will pay 900 points as the cost of investment. The 10 unused tokens will be converted to 100 points. Your payoff will be $1600-900+100=800$ points if you win, and $-900+100=-800$ if you lose.

Though your points will automatically be updated to reflect the amount of the payoff, you must understand how your payoff is determined. Three questions on the screening test will ask you to calculate the payoff in hypothetical situations.

\section{Other information:}

Your virtual competitors will make their own best possible decisions given their environment, and will assume that you will make your best decisions on investment choices in your chosen environment. That is, their decision strategies are preset; they will not adjust their decisions based on your investment. At the end of the round, you will be informed whether you have won and how much you have earned (or lost). After you begin with 7,000 points in round 1, your points in each new round will be cumulative. Please note that the cost of investment cannot exceed the cumulative points you currently have. For example, if you have 400 points now, you cannot invest more than 20 tokens, because the square of 20 is 400 .

Now you will be given the screening test.

\section{Screening Test:}


You will answer six multiple choice questions. You can participate in the experiment only if ALL of your answers are correct. If you do not pass the screening test, you will be asked to leave without payment. The main purpose of this screening test is to help you understand the instructions, not to cause you any stress. It is okay for you to ask an experimenter to help you if you are in doubt.

Q1 The experiment will consist of (A) rounds. In each round, you will have (B) tokens. You will start with $(\mathrm{C})$ points. What are the numbers $(\mathrm{A}),(\mathrm{B})$ and $(\mathrm{C})$ that will make these statements true?

Q2 Suppose you have 22,000 points at the end of the experiment. How much will you get paid in cash?

Q3 Suppose you choose an environment described by the range $[101.78,200.30]$. Which of the following numbers CANNOT be your environmental support: 144.34, 199.00, 103.55, $204.23 ?$

Q4 Assume that in the first round you invest 0 tokens and you win. What will your payoff be?

Q5 Assume that in the first round you invest 20 tokens and you lose. What will your payoff be?

Q6 Assume that in the first round you invest 14 tokens and you win. What will your payoff be?

[Let those who fail the screening test leave. The experiment begins.]

[The experiment ends. After filling out a short survey form, subjects leave with payments.]

\section{References}

Agranov, M., And C. Tergiman (2013): "Incentives and Compensation Schemes: An Experimental Study," International Journal of Industrial Organization, 31(3), 238-247.

Akerlof, G. A., And R. E. Kranton (2005): "Identity and the Economics of Organizations," The Journal of Economic Perspectives, 19(1), 9-32.

Allard, R. J. (1988): "Rent-Seeking with Non-Identical Players," Public Choice, 57(1), 3-14. 
Amann, E., And W. Leininger (1996): "Asymmetric All-Pay Auctions with Incomplete Information: The Two-Player Case," Games and Economic Behavior, 14(1), 1-18.

Andrade, E. B., And G. Iyer (2009): "Planned Versus Actual Betting in Sequential Gambles," Journal of Marketing Research, 46(3), 372-383.

Bull, C., A. Schotter, And K. Weigelt (1987): "Tournaments and Piece Rates: An Experimental Study," Journal of Political Economy, 95(1), 1-33.

Dechenaux, E., D. Kovenock, And R. M. Sheremeta (2014): "A Survey of Experimental Research on Contests, All-Pay Auctions and Tournaments," Experimental Economics, pp. $1-61$.

Dubey, P. (2013): "The Role of Information in Contests," Economics Letters, 120(2), 160-163.

Frank, R. (2016): Success and Luck: Good Fortune and the Myth of Meritocracy. Princeton University Press.

Gale, D., And L. S. Shapley (1962): "College Admissions and the Stability of Marriage," The American Mathematical Monthly, 69(1), 9-15.

Gilpatric, S. M. (2009): "Risk Taking in Contests and the Role of Carrots and Sticks," Economic Inquiry, 47(2), 266-277.

Gneezy, U., And R. Smorodinsky (2006): "All-Pay Auctions-An Experimental Study," Journal of Economic Behavior \& Organization, 61(2), 255-275.

Harbring, C., and B. Irlenbusch (2011): "Sabotage in Tournaments: Evidence from a Laboratory Experiment," Management Science, 57(4), 611-627.

Hvide, H. K. (2002): "Tournament Rewards and Risk Taking," Journal of Labor Economics, 20(4), pp. 877-898.

ImAs, A. (2014): "The Realization Effect: Risk-Taking after Realized versus Paper Losses," SSRN Working Paper Series.

KamiJo, Y. (2016): "Rewards versus Punishments in Additive, Weakest-Link, and Best-Shot Contests," Journal of Economic Behavior \& Organization, 122, 17-30. 
KirkegaARD, R. (2013): "Handicaps in Incomplete Information All-Pay Auctions with a Diverse Set of Bidders," European Economic Review, 64, 98-110.

KrÄKel, M., AND D. Sliwka (2004): "Risk Taking in Asymmetric Tournaments," German Economic Review, 5(1), 103-116.

Krishna, V., And J. Morgan (1997): "An Analysis of the War of Attrition and the All-Pay Auction," Journal of Economic Theory, 72(2), 343-362.

(1998): "The Winner-Take-All Principle in Small Tournaments," Advances in Applied Microeconomics, Vol 7 (M. Baye, Ed.), Stamford: JAI Press, 1998.

Langer, T., And M. Weber (2008): "Does Commitment or Feedback Influence Myopic Loss Aversion?: An Experimental Analysis," Journal of Economic Behavior 63 Organization, 67(3-4), 810-819.

Lazear, E. P., And S. Rosen (1981): "Rank-Order Tournaments as Optimum Labor Contracts," Journal of Political Economy, 89(5), 841-864.

Moldovanu, B., And A. Sela (2001): "The Optimal Allocation of Prizes in Contests," The American Economic Review, 91(3), 542-558.

Moldovanu, B., A. Sela, And X. Shi (2012): "Carrots and Sticks: Prizes and Punishments in Contests," Economic Inquiry, 50(2), 453-462.

Nieken, P. (2010): "On the Choice of Risk and Effort in Tournaments-Experimental Evidence," Journal of Economics 83 Management Strategy, 19(3), 811-840.

Nti, K. O. (1999): "Rent-Seeking with Asymmetric Valuations," Public Choice, 98, 415-430.

Parreiras, S. O., And A. Rubinchik (2010): "Contests with Three or More Heterogeneous Agents," Games and Economic Behavior, 68(2), 703-715.

Pérez-Castrillo, D., And D. Wettstein (2014): "Innovation Contests," CESifo Working Paper Series.

Schotter, A., And K. Weigelt (1992): "Asymmetric Tournaments, Equal Opportunity Laws, and Affirmative Action: Some Experimental Results," The Quarterly Journal of Economics, 107(2), 511-539. 
Siegel, R. (2009): "All-Pay Contests," Econometrica, 77(1), 71-92.

Stein, W. E. (2002): "Asymmetric Rent-Seeking with More than Two Contestants," Public Choice, 113, 325-336.

Tullock, G. (1980): "Efficient Rent-Seeking," Toward a Theory of the Rent Seeking Society, (J. Buchanan, R. Tollison, and G. Tullock, Eds.) College Station: Texas A\&M University Press, 97-112.

Weber, M., And H. Zuchel (2005): "How Do Prior Outcomes Affect Risk Attitude? Comparing Escalation of Commitment and the House-Money Effect," Decision Analysis, 2(1), $30-43$. 